ACCEPTED for Publication in The Astrophysical Journal

Preprint typeset using LATEX style emulateapj v. 01/23/15

\title{
A GIANT GREEN PEA IDENTIFIED IN THE SPECTROSCOPY OF SPATIALLY EXTENDED [O III] SOURCES
}

\author{
Suraphong Yuma $^{1}$, Masami Ouchi ${ }^{2}$, Seiji Fujimoto ${ }^{2,3}$, TAKashi Kojima ${ }^{2,4}$, And Yuma Sugahara ${ }^{2,4}$ \\ ${ }^{1}$ Department of Physics, Faculty of Science, Mahidol University, Bangkok 10400, Thailand; suraphong.yum @ mahidol.ac.th \\ ${ }^{2}$ Institute for Cosmic Ray Research, The University of Tokyo, Kashiwa-no-ha, Kashiwa 277-8582, Japan \\ ${ }^{3}$ Department of Astronomy, University of Tokyo, Hongo, Bunkyo-ku, Tokyo 113-0033, Japan and \\ ${ }^{4}$ Department of Physics, University of Tokyo, Hongo, Bunkyo-ku, Tokyo 113-0033, Japan \\ Accepted for Publication in The Astrophysical Journal
}

\begin{abstract}
We present the results of the deep Subaru/FOCAS and Keck/MOSFIRE spectroscopy for four spatially extended [O III] $\lambda \lambda 4959,5007$ sources, dubbed [O III] blobs, at $z=0.6-0.8$ that are originally pinpointed by large-area Subaru imaging surveys. The line diagnostics of the rest-frame optical lines suggests that only one [O III] blob, OIIIB-3, presents an AGN signature, indicating that hot gas of the rest of the [O III] blobs is heated by star formation. One of such star-forming [O III] blobs, OIIIB-4, at $z=0.838$ has an [O III] equivalent width of $845 \pm 27 \AA$ and an [O III] to [O II] $\lambda \lambda 3726,3729$ ratio of $[\mathrm{O} \mathrm{III}] /[\mathrm{O} \mathrm{II}]=6.5 \pm 2.7$ that are as high as those of typical green peas (Cardamone et al. 2009). The spatially resolved spectrum of OIIIB-4 shows $[\mathrm{O} \mathrm{III}] /[\mathrm{O} \mathrm{II}]=5-10$ over $14 \mathrm{kpc}$ in the entire large [O III] extended regions of OIIIB-4, unlike the known green peas whose strong [O III] emission region is compact. Moreover, OIIIB-4 presents no high-ionization emission lines unlike green beans that have extended [O III] emission with a type-2 AGN. OIIIB-4 is thus a giant green pea, which is a low stellar mass $\left(7 \times 10^{7} M_{\odot}\right)$ galaxy with a very high specific star-formation rate $($ sSFR $\left.=2 \times 10^{2} \mathrm{Gyr}^{-1}\right)$, a high ionization parameter $\left(q_{\text {ion }} \sim 3 \times 10^{8} \mathrm{~cm} \mathrm{~s}^{-1}\right)$, and a low metallicity similar to those of green peas. Neither an AGN-light echo nor a fast radiative shock likely takes place due to the line diagnostics for spatially-resolved components of OIIIB-4 and no detections of He II $\lambda 4686$ or [Ne V] $\lambda 3346,3426$ lines that are fast-radiative shock signatures. There is a possibility that the spatially-extended [O III] emission of OIIIB-4 is originated from outflowing gas produced by the intense star formation in a density-bounded ionization state. Keywords: galaxies: high redshift — galaxies: evolution — galaxies: formation - ISM: jets and outflows
\end{abstract}

\section{INTRODUCTION}

A galaxy is not a closed box that can evolve itself without exchanging materials with the environment. The accretion of cooled gas onto the galaxy is important for the galaxy to form new stars in the $\Lambda$ Cold Dark Matter $(\Lambda \mathrm{CDM})$ model. However, the inflow alone would cause an overestimation of star formation at both low-mass and high-mass ends (e.g., Bell et al. 2003; Mutch et al. 2013). The feedback mechanism involving gas outflows becomes a default tool to resolve the discrepancy in hydrodynamic simulations (e.g., Benson et al. 2003, Somerville et al. 2008; Oppenheimer et al. 2010; van de Voort et al. 2011). The evolutional state of a galaxy is thus thought to depend on the balance of the gas flows in and out of the galaxy (e.g., Lilly et al. 2013). In addition, the galacticscale outflow is considered a solution for various observational phenomena including regulating the main sequence of galaxies on the mass-SFR (star formation rate) diagram (e.g., Noeske et al. 2007) and the mass-metallicity relation (e.g., Tremonti et al. 2004), and enriching the chemical abundance of interstellar medium (ISM) and intergalactic medium (IGM; e.g., Martin 2005: Rupke et al. 2005a b; Weiner et al. 2009. Coil et al. 2011).

Outflows have been massively studied in several types of galaxies ranging from normal star-forming galaxies (e.g., Weiner et al. 2009, Steidel et al. 2010; Erb et al. 2012, Martin et al. 2012; Bradshaw et al. 2013; Rubin et al. 2014) and submillimeter galaxies (SMG; e.g., Alexander et al.|2010) to more extreme systems like radio galaxies (e.g., Nesvadba et al. 2008; Liu et al. 2013), ultra luminous infrared galaxies (ULIRGs, e.g., Heckman et al. 1990; Martin 2005; Rupke et al. 2005a b; Soto et al. 2012), and active galactic nuclei
(AGNs; e.g., Cicone et al. 2014, Cheung et al. 2016, Rupke et al. 2017). Outflows are found to be ubiquitous in galaxies with the SFR surface density larger than $\sim 0.1 M_{\odot} \mathrm{yr}^{-1} \mathrm{kpc}^{-2}$ and become stronger in more massive star-forming galaxies with higher SFRs (Heckman et al. 2000; Martin 2005; Weiner et al. 2009, Kornei et al.2012; Martin et al.2012). In the case of AGNs, the outflows strongly correlate with the black hole mass $\left(M_{\mathrm{BH}}\right)$ in that the mass outflow rate increases with increasing $M_{\mathrm{BH}}$ (Rupke et al. 2017). An active supermassive black hole alone could fuel the large-scale outflow even in a low-luminosity AGN (Cheung et al.2016).

Most of these studies are based mainly on the optical/nearinfrared spectroscopic observations of the blueshifted interstellar absorption lines such as $\mathrm{Na} \mathrm{I} \lambda \lambda 5890,5896$, $\operatorname{Mg}$ II $\lambda \lambda 2796,2803$, Fe II $\lambda 2374$, and Fe II $\lambda 2383$ to indicate the outflow signature. No systematic search had been done by using only the imaging data until Yuma et al. (2013) proposed to use the narrowband technique to systematically identify the gas outflowing galaxies by selecting star-forming galaxies with the strong [O II] $\lambda \lambda 3726,3729$ emission line spatially extended over $30 \mathrm{kpc}$ beyond the stellar components. They called this [O II] extended object "[O II] blob" or in short "O IIB." The spatial extension of a metal line like the [O II] emission line that is redshifted and falls into the narrowband image might indicate a large-scale outflow beyond the galaxy rather than an evidence of a gas inflow from the metal-poor IGM (e.g., Aguirre et al. 2008, Fumagalli et al. 2011). Yuma et al. (2013) successfully identified twelve O IIBs at $z \sim 1.2$, one of which is classified as an obscured AGN. The others are potentially normal star-forming galaxies as they are not detected in X-ray or radio $1.4 \mathrm{GHz}$ wavelengths. The hypothesis that the extended [O II] emission line may represent the 
outflow from the [O II] blobs is spectroscopically confirmed by the traditional method of detecting blueshifted interstellar absorption lines (Yuma et al. 2013, Harikane et al. 2014). The systematic search was then expanded toward lower and higher redshifts; i.e., $z=0.1-1.5$ (Yuma et al. 2017). In Yuma et al. (2017), the spatially extended emission lines include [O II $] \lambda \lambda 3726,3729$, [O III] $\lambda 5007$, and $\mathrm{H} \alpha \lambda 6563$. The resulting sample is named accordingly as an [O II] blob (O IIB), an [O III] blob (O IIIB), and an $\mathrm{H} \alpha$ blob (HAB). They found 77 blobs at $z=0.4-1.5$ in total, eight of which are likely to be powered by AGNs. It is suggested that the blobs are mostly normal star-forming galaxies. It is of interest to investigate an energy source for the extended emission lines of the blobs that are identified as normal star-forming galaxies.

Galaxies with large extension of emission lines have recently been discovered by many studies. Lin et al. (2017) discovered a giant $\mathrm{H} \alpha$ blob with the $\mathrm{H} \alpha$ extent of $3-4 \mathrm{kpc}$ in radius. The spatial extension of the [O III] emission line is also found (Brammer et al. 2013, Sun et al.2017). An AGN is thought to be a primary source of energy to produce the largescale outflow in both the $\mathrm{H} \alpha$ blob discovered by Lin et al. (2017) and the [O III] blobs studied by Sun et al. (2017). The latter work suggested that the size of [O III] extension strongly correlates with the AGN luminosity. Sun et al. (2017) also argued that there is no AGN luminosity threshold for launching the outflow, which is consistent with Cheung et al. (2016). It means that it is common to see the extended emission line in all AGNs regardless of their luminosities.

Although there are certain detailed studies about the extended emission lines in the normal star-forming galaxies (e.g., Genzel et al. 2011; Newman et al. 2012a b), the physical mechanism responsible for the spatially extended emission lines beyond the stellar component of the galaxies is still not well understood. To date, many studies about the ISM of galaxies are conducted with the integral field unit (IFU) observations (e.g., Förster Schreiber et al. 2009, 2014, Lin et al. 2017; Sun et al. 2017). This three-dimensional (3D) imaging spectrograph enables researchers to investigate the spectra of the entire galaxies simultaneously with $2 \mathrm{D}$ imaging. In this paper, instead of using the IFU, we take a simple step by observing the [O III $]$ blobs at $z=0.63$ and $z=0.83$ that show no AGN signature in X-ray and radio wavelengths originally selected by Yuma et al. (2017) with a spectrograph in the multiobject spectroscopy mode. By designing the slit direction to cover the longest extension of the emission lines of the blobs, we are able to determine the emission-line ratios and examine the physical properties of the extended component of the emission lines along the slit direction.

In section 2, we explain about the [O III]-blob targets for the spectroscopic observations. Section 3 describes details of both optical and near-infrared observations and data reduction processes. We then report the results of systematic redshifts, outflow signature, AGN contribution, and properties of the ISM including the radial profile of the [O III] blobs in Section 4 . We also report the discovery of a giant green pea in this section. In section 5, we discuss the plausible scenarios that could be responsible for the spatial extension of the emission lines seen in the [O III] blobs. The last section, Section 6 is the summary of all our findings. Throughout this paper, we adopt the standard $\Lambda$ CDM cosmology with $H_{0}=70$ $\mathrm{km} \mathrm{s}^{-1} \mathrm{Mpc}^{-1}, \Omega_{m}=0.3$, and $\Omega_{\lambda}=0.7$. All magnitudes are given in the AB system (Oke \& Gunn 1983).

\section{TARGETS FOR SPECTROSCOPY}
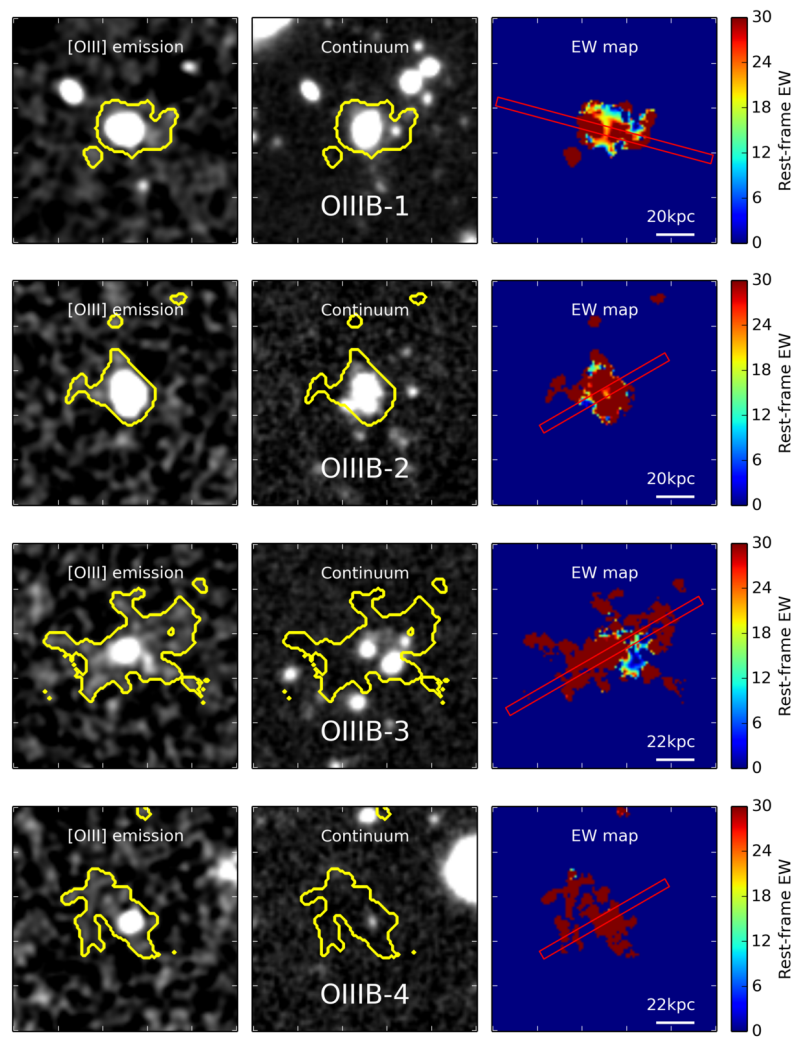

Figure 1. From left to right: the emission-line $(N B-B B)$, stellar continuum, and equivalent-width maps of 4 [O III] blobs at $z=0.63$ and $z=0.83$, respectively. The emission-line images are $N B 816-R z$ and $N B 921-z^{\prime}$ for the blobs at $z=0.63$ and $z=0.83$, respectively. $R z$ is defined as $R z$ $\equiv\left(R+2 z^{\prime}\right) / 3$. IDs are indicated in the middle panel. North is up and East is to the left. Yellow contours in the left and middle panels indicate the isophotal area of the emission-line flux measured down to $1.2 \times 10^{-18}$ $\mathrm{erg} \mathrm{s}^{-1} \mathrm{~cm}^{-2} \operatorname{arcsec}^{-2}$. The color bar in the right panel corresponds to the rest-frame equivalent width per pixel. The magenta rectangle shows the slit direction that we used to observe the spectrum of the [O III] blobs with Subaru/FOCAS.

We conduct the spectroscopic observations of four [O III] blobs at $z \sim 0.63$ and $z \sim 0.83$. Our spectroscopic sample is originally identified as an [O III] blob by Yuma et al. (2017). They conducted the systematic survey of [O II], [O III], and $\mathrm{H} \alpha$ blobs at $z=0.1-1.5$; i.e., star-forming galaxies with strong [O II], [O III], and $\mathrm{H} \alpha$ emission lines, respectively, spatially extended over $30 \mathrm{kpc}$. The emission-line extension is measured down to surface flux limit of $1.2 \times 10^{-18}$ $\mathrm{erg} \mathrm{s}^{-1} \mathrm{~cm}^{-2} \operatorname{arcsec}^{-2}$. Among 77 blobs at $z=0.1-1.5$ found by Yuma et al. (2017), there are 4 and 13 [O III] blobs at $z=0.63$ and $z=0.83$, respectively. All blobs are crossmatched with the X-ray and radio catalogs to primarily check for the AGN existence. The X-ray source catalog is obtained from Ueda et al. (2008). It is observed with the European Photon Imaging Camera (Strüder et al. 2001; Turner et al. 2001) on the XMM-Newton telescope (Jansen et al. 2001). The flux limits of the catalog are $6 \times 10^{-16}, 8 \times 10^{-16}, 3 \times 10^{-15}$, and $5 \times 10^{-15} \mathrm{erg} \mathrm{s}^{-1} \mathrm{~cm}^{-2}$ in the $0.5-2,0.5-4.5,2-10$, and $4.5-10 \mathrm{keV}$ bands, respectively. These flux limits approximately correspond to the X-ray luminosities of $10^{42} \mathrm{erg} \mathrm{s}^{-1}$ at $z \sim 0.7$. The radio catalog is from Simpson et al. (2006). The radio data are observed with the Very Large Array (VLA) in $1.4 \mathrm{GHz}$ down to the limit of $100 \mu \mathrm{Jy}$. All of the [O III] blobs are not detected in X-ray and radio wavelengths.

The criteria of the spectroscopic targets are that 1) they 
Table 1

Photometric properties of [O III] blobs at $z=0.63$ and $z=0.83$ for spectroscopic follow-up

\begin{tabular}{cccccc}
\hline \hline Object Name & $\alpha$ (J2000) & $\delta$ (J2000) & $\begin{array}{c}N B-B B \\
(\mathrm{mag})\end{array}$ & $\begin{array}{c}L\left([\mathrm{O} \text { III] })^{\mathrm{a}}\right. \\
\left(10^{41} \mathrm{erg} \mathrm{s}^{-1}\right)\end{array}$ & $\begin{array}{c}\text { Isophotal Area } \\
\left(\mathrm{kpc}^{2}\right)\end{array}$ \\
\hline \multicolumn{5}{c}{ [O III] blobs at $z=0.63$} \\
\hline O IIIB-1 (OIIIB063s-1) & 021750.244 & -050004.159 & $22.04 \pm 0.14$ & $4.99 \pm 0.7$ & 1121.2 \\
O IIIB-2 (OIIIB063s-2) & 021905.902 & -051348.599 & $22.27 \pm 0.15$ & $4.04 \pm 0.7$ & 1121.2 \\
\hline \multicolumn{7}{c}{ [O III] blobs at $z=0.83$} \\
\hline O IIIB-3 (OIIIB083m-2) & 021903.734 & -051153.243 & $22.77 \pm 0.13$ & $4.40 \pm 0.6$ & 1675.0 \\
O IIIB-4 (OIIIB083m-12) & 021858.701 & -051258.126 & $23.32 \pm 0.23$ & $2.64 \pm 0.6$ & 981.9
\end{tabular}

Note:

a The luminosity of the [O III] emission lines is derived from the magnitude in $N B-B B$ and is not corrected for internal dust attenuation.

are among the $[\mathrm{O} I I I]$ blobs with the largest extension of the [O III] emission line and/or 2) they are located close to each other so that we can maximize the number of the [O III] blobs observed within one mask. As a result, we select 4 [O III] blobs as the spectroscopic targets and observe them with two masks. The targets are OIIIB063s-1, OIIIB063s2, OIIIB083m-2, and OIIIIB083m-12. The IDs are from the original catalog by Yuma et al. (2017). O IIIB indicates that it is an [O III] blob, a galaxies with the extended [O III] emission line. Three numbers after O IIIB represent the redshifts; for example, 063 means the blob is at $z=0.63$. The letter "s" or " $\mathrm{m}$ " stands for single or multiple, respectively, indicating the number of stellar components in one blob. The last numbers show the order of blobs starting from " 1 " for the blob with the largest extension of the emission line. To avoid any confusion, we hereafter refer to OIIIB063s-1, OIIIB063s2, OIIIB083m-2, and OIIIIB083m-12 as O IIIB-1, O IIIB-2, O IIIB-3, and O IIIB-4, respectively. Figure 1 shows the 2dimensional (2D) images of the [O III] emission line, the stellar continuum, and the equivalent-width (EW) map of our 4 targets. The emission-line images $(N B-B B)$ correspond to the $N B 816-R z$ and $N B 921-z^{\prime}$ images for the [O III] blobs at $z=0.63$ and $z=0.83$, respectively, where $R z$ is defined as $R z \equiv\left(R+2 z^{\prime}\right) / 3$. The contour of the [O III] extension is also displayed in the figure. The slit direction with the exact width and length is shown in the right panel of Figure 1 Coordinates and photometric properties of these four [O III] blobs derived by Yuma et al. (2017) are listed in Table 1

\section{OBSERVATION AND DATA REDUCTION}

\subsection{Subaru/FOCAS}

We conducted the optical spectroscopic follow-up observations with Subaru/Faint Object Camera and Spectrograph (FOCAS; Kashikawa et al. 2002) on October 21-22, 2014 and December 2-4, 2015 (S14B-130 and S15B-059; PI: S. Yuma). Details about the Subaru/FOCAS spectroscopic observations are described in Yuma et al. (2017). Briefly speaking, we performed the observations in the multi-object spectroscopy (MOS) mode with the VPH450 grating and the VPH850 grating with the SO58 order-cut filter. The gratings cover $3800-5250 \AA$ with a dispersion of $0.37 \AA /$ pixel and $5800-10500 \AA$ with $1.17 \AA$ A pixel, respectively. We adopted the slit width of $0 . \prime 8$ providing the spectral resolutions of 1700 and 750 for VPH450 and VPH850+SO58, respectively. We used 2 MOS masks to observed 4 [O III] blobs. Mask1 contains only O IIIB-1 which is the largest [O III] blob
Table 2

Details of optical and near-infrared spectroscopic observations for 4 [O III] blobs at $z=0.63$ and $z=0.83$

\begin{tabular}{lcc}
\hline \hline \multicolumn{1}{c}{ Ins./Grating+Filter } & $\begin{array}{c}\text { Exposure time } \\
\text { (minutes) }\end{array}$ & $\begin{array}{c}3 \sigma \text { flux limit } \\
\left(\mathrm{erg} \mathrm{s}^{-1} \mathrm{~cm}^{-2}\right)\end{array}$ \\
\hline Mask1: O IIIB-1 & & \\
\hline FOCAS/VPH450 & 180 & $\simeq 3.8 \times 10^{-17}$ \\
FOCAS/VPH850+SO58 & 120 & $\simeq 1.1 \times 10^{-18}$ \\
MOSFIRE/Y & 285 & $\simeq 3.1 \times 10^{-18}$ \\
\hline Mask2: O IIIB-2, O IIIB-3, and O IIIB-4 & \\
\hline VPH450 & 260 & $\simeq 1.1 \times 10^{-17}$ \\
VPH850+SO58 & 100 & $\simeq 1.0 \times 10^{-18}$ \\
\hline
\end{tabular}

Note:

${ }^{a}$ For O IIIB-2, we discard two observed frames to avoid the cosmic ray at the wavelength of the $\mathrm{H} \beta \lambda 4861$ emission line. Therefore, the $3 \sigma$ flux limit of O IIIB-2 is $\simeq 3.7 \times 10^{-18} \mathrm{erg} \mathrm{s}^{-1} \mathrm{~cm}^{-2}$.

at $z=0.63$, while the other 3 blobs (O IIIB-2, O IIIB-3, and O IIIB-4) are so close to each other that we can place them all in Mask2. The on-source exposure times for Mask1 are 180 minutes and 120 minutes in VPH450 and VPH850+SO58, respectively. For Mask2, the exposure times are 260 minutes and 100 minutes. The sky when we observed the target in Mask1 was quite clear in that we obtain the seeing size of $\sim 0 .{ }^{\prime \prime} 6-0 . \prime 7$. Unfortunately, the weather was getting worse when we observed Mask2. The seeing size is roughly $\sim 0$. . 9 . We carefully designed the slit direction to cover the longest extent of the [O III] emission line as shown with the magenta color in Figure 1. Note that, in Mask2, we intended to do so with O IIIB-3, which is the second largest [O III] blob at $z=0.83$. Because all slits in one mask are required to arrange in the identical direction, the slit directions for O IIIB-2 and $\mathrm{O}$ IIIB-4 are consequently not oriented to the longest extension of their [O III] emission lines.

Data reduction has been carried out with the FOCASRED package, which is the specific pipeline on Imaging Reduction and Analysis Facility (IRAF) for Subaru/FOCAS data reduction. We started with bias subtraction, flat fielding, and distortion correction. Wavelength calibration is then performed by using the ThAr lamp and $\mathrm{OH}$ airglow emission lines for VPH450 and VPH850+SO58 spectra, respectively. After we subtracted the sky background, we stacked the spectra and extracted them along the spatial direction to create one-dimensional (1D) spectra. The extracting width is de- 
signed to cover the entire spatial extension of the emission line. Flux calibration is carried out by using the standard star G191-B2B observed with the same slit width. It is extracted to $1 \mathrm{D}$ spectrum with the same extraction width as the target. The slit loss is automatically corrected during the flux calibration process. The $3 \sigma$ flux limits are summarized in Table 2 .

\subsection{Keck/MOSFIRE}

Near-infrared (NIR) observation was conducted only for O IIIB-1 with Multi-Object Spectrometer For Infrared Exploration (MOSFIRE; McLean et al. 2012) attached on Keck I telescope. O IIIB-1 was observed as a filler target with the Yband filter on January 3-4, 2014 (S15B-075; PI: M. Ouchi). The main objective of the MOSFIRE proposal is to confirm the Lyman alpha emitters at $z \sim 5$.7. The spectral resolution is 3388 with the standard $0 . \prime 7$ slit width. The total exposure time is 4.45 hours with seeing size of $\sim 0{ }^{\prime \prime} 7$. Data reduction is performed with MOSFIRE data reduction pipeline ${ }^{1}$ following the standard processes for reducing the spectra and flux calibration with the standard stars taken during the observations. The exposure time and the limiting flux are also listed in Table 2.

\section{RESULTS}

\subsection{Systemic redshifts}

Before we investigate the spectra in details, we first check whether our [O III] blob sample is at the targeted redshifts. We examine the emission lines detected in the VPH850+SO58 spectra. The [O III] blobs at $z \sim 0.63$ and $z \sim 0.83$ are originally identified by the narrowband technique using the NB816 and NB921 images, respectively. So we expect the [O III] emission line to be around $8090-8210 \AA$ for O IIIB1 and O IIIB-2 and 9130 - 9262 $\AA$ for O IIIB-3 and O IIIB-4. In fact, Yuma et al. (2017) already confirmed that the emission line detected in the wavelength ranges mentioned above is [O III] $\lambda 5007$ based on the spectroscopic confirmation of [O II] $\lambda 3727, \mathrm{H} \beta \lambda 4861$, and [O III] $\lambda 4959$ emission lines at the corresponding wavelengths. They confirmed that O IIIB-1 and $\mathrm{O} I \mathrm{IIB}-2$ are at $z=0.621$ and at $z=0.641$, respectively, whereas O IIIB-3 and O IIIB-4 are both at $z=0.838$. We fit the $[\mathrm{O}$ II $], \mathrm{H} \beta$, and [O III] emission lines with a single Gaussian profile and adopt the center of the Gaussian profile that is best fitted to the [O III] $\lambda 5007$ emission line as the spectroscopic redshift for each [O III] blob (Table 3). Our resulting spectroscopic redshifts are consistent with those derived by Yuma et al. (2017).

\subsection{Spectroscopic Properties of Emission lines}

The Subaru/FOCAS spectra of 4 [O III] blobs at $z \sim 0.63-$ 0.83 are illustrated in Figure 2 at the observed wavelengths of the [O II] $\lambda 3727, \mathrm{H} \beta \lambda 4861$, and [O III] $\lambda \lambda 4959,5007$ emission lines. In each plot, we show the two-dimensional spectral image in the top panel and the one-dimensional spectrum in the bottom one. All the lines for a given blob are plotted with the same flux scale to provide the idea of the strength of the emission lines as compared to one another. The top row of Figure 2 shows that the stellar continuum is significantly detected in O IIIB-1. The excessive extension of the emission lines beyond the stellar components is clearly seen in the 2D spectral image.

\footnotetext{
${ }^{1}$ https://keck-datareductionpipelines.github.io/MosfireDRP/
}

Table 3

Spectroscopic redshifts and dust-corrected fluxes of [O II], $\mathrm{H} \beta$, and [O III] emission lines for 4 [O III] blobs at $z=0.63-0.83$

\begin{tabular}{ccrrr}
\hline \hline Target & \multirow{2}{*}{$z_{\text {spec }}$} & \multicolumn{2}{c}{ Observed Flux $\left(\times 10^{-16} \mathrm{erg} \mathrm{s}^{-1} \mathrm{~cm}^{-2}\right)$} \\
& & \multicolumn{1}{c}{$[\mathrm{O}$ II $]$} & \multicolumn{1}{c}{ [O III] } & \multicolumn{1}{c}{} \\
\hline O IIIB-1 & 0.6210 & $16.67 \pm 0.29$ & $5.02 \pm 0.27$ & $12.16 \pm 0.25$ \\
O IIIB-2 & 0.6413 & $7.33 \pm 0.60$ & $1.53 \pm 0.31$ & $3.58 \pm 0.65$ \\
O IIIB-3 & 0.8365 & $6.12 \pm 0.51$ & $0.71 \pm 0.35$ & $5.48 \pm 0.68$ \\
O IIIB-4 & 0.8379 & $0.31 \pm 0.11$ & $0.30 \pm 0.12$ & $2.02 \pm 0.12$ \\
\hline
\end{tabular}

Although we marginally detect the stellar continuum of O IIIB-2, the emission lines of O IIIB-2 seem to be extended over the stellar continuum in the spatial direction. Likewise, we are able to see the spatial extension of the [O III] $\lambda 5007$ emission line of O IIIB-3. O IIIB-3 shows the significantly larger spectral line width of the [O III] $\lambda 5007$ emission as compared with O IIIB-1 and O IIIB-2. The full width at half maximum (FWHM) of the [O III] $\lambda 5007$ line of O IIIB-3 is $419.5 \pm 76.8 \mathrm{~km} \mathrm{~s}^{-1}$ after correcting for the instrument, while it is in the order of $150-200 \mathrm{~km} \mathrm{~s}^{-1}$ for O IIIB-1 and O IIIB2 . For O IIIB-4, the stellar continuum is unfortunately not detected, but we can detect the [O III] emission line with the high signal-to-noise $(\mathrm{S} / \mathrm{N})$ ratio. However, the $[\mathrm{O} \mathrm{II}]$ and $\mathrm{H} \beta$ emission lines are marginally detected with the $\mathrm{S} / \mathrm{N}$ ratios of $2-3 \sigma$. The observed flux of each emission line is derived by fitting the 1D spectrum with a single Gaussian profile and corrected for the dust attenuation, which is explained in Appendix A. In short, we derive the dust attenuation of O IIIB-1 and O IIIB-2 from the Balmer decrement using the $\mathrm{H} \gamma / \mathrm{H} \beta$ line ratios. We find that the color excesses $(E(B-V))$ estimated from the Balmer decrement is in agreement with those derived by fitting the observed spectral energy distribution of the blob with the stellar population synthesis models (SED fitting method; Appendix A. Therefore, for O IIIB-3 and O IIIB-4 whose $\mathrm{H} \gamma$ emission lines are not detected, we adopt the color excesses derived by the SED fitting method. The nebular color excesses of O IIIB-3 and O IIIB-4 are then calculated by using the relation between the nebula and the stellar dust extinctions in Calzetti et al. (2000). More details on the SED fitting procedures of the [O III] blobs are explained in Section 4.7 and Yuma et al. (2017). We summarize the dust-corrected fluxes of the [O II] $\lambda 3727, \mathrm{H} \beta \lambda 4861$, and [O III] $\lambda 5007$ emission lines with corresponding $1 \sigma$ uncertainties in Table 3 The extended features seen in O IIIB-1, O IIIB-2, and O IIIB-3 are just confirmed that the blob selection method by Yuma et al. (2017) is efficient in selecting galaxies with the spatially extended emission line.

\subsection{Outflow Signature}

The galaxies with spatially extended emission lines like [O III] blobs are thought to be in an ongoing process of the large-scale outflow. The gas outflow is already confirmed in [O II] blobs at $z \sim 1.2$ with Subaru/FOCAS, VLT/VIMOS, and Magellan/LDSS spectra of the [O II] blobs at $z \sim 1.2$ (Yuma et al. 2013; Harikane et al. 2014); however, this is the first time to confirm if the [O III] blobs are in the middle of the outflow process. The blueshifted interstellar absorption lines are one of the most common methods that are used to study the outflow process of the galaxies. Unfortunately, the spectra of three out of four blobs in the VPH450 grating, in which we expect to detect the absorption lines, have too low $\mathrm{S} / \mathrm{N}$ ratios. We only detect the absorption lines in the O IIIB-1 spectrum. Figure 3 shows the blueshifted Fe II $\lambda 2600$ absorp- 

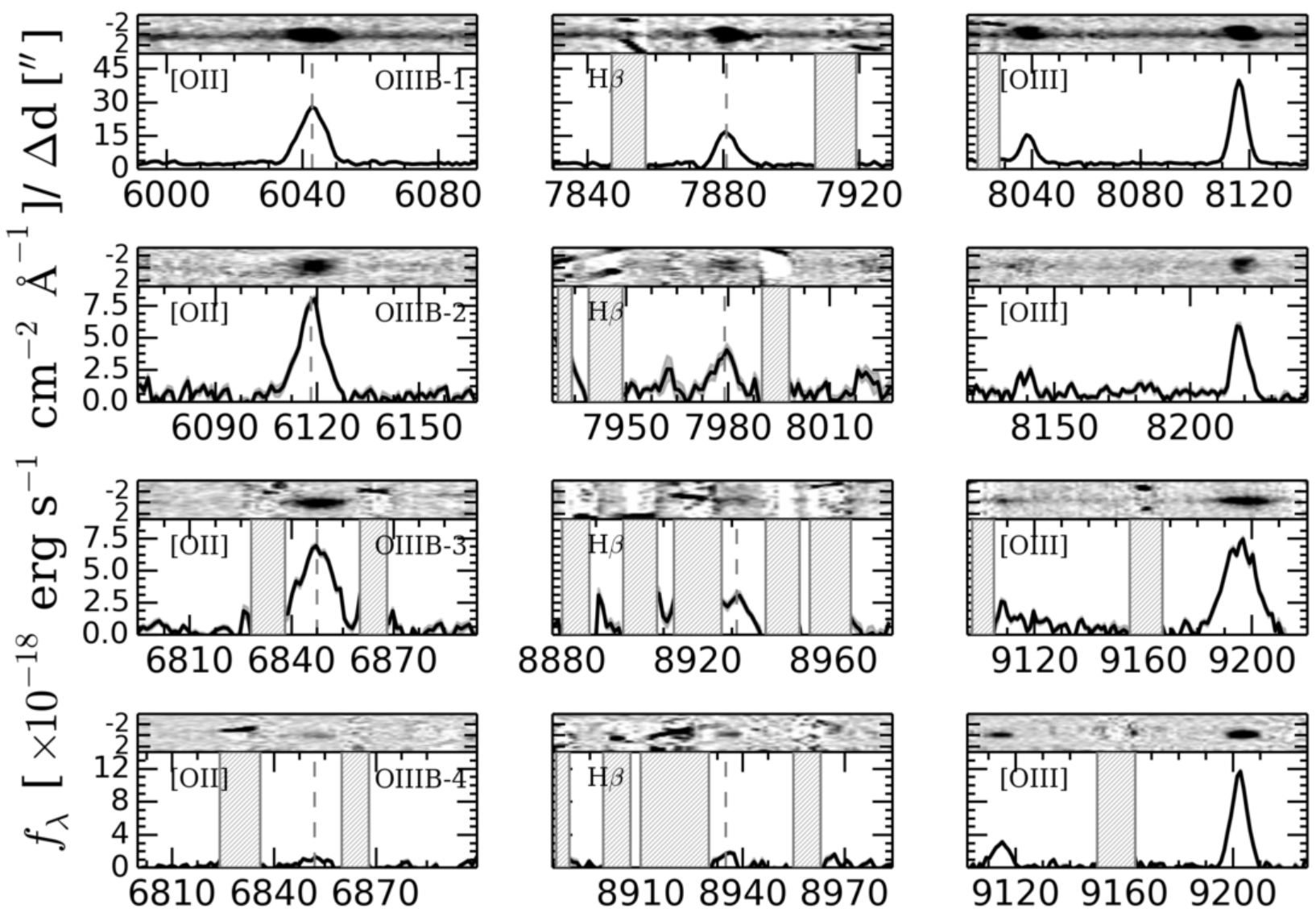

Observed Wavelength $[\AA ̊]$

Figure 2. Subaru/FOCAS spectra of O IIIB-1, O IIIB-2, O IIIB-3, and O IIIB-4 from the top to the bottom panels, respectively. The left, middle, and right columns show the $[\mathrm{O} \mathrm{II}], \mathrm{H} \beta$, and $[\mathrm{O} \mathrm{III}]$ emission lines of each object, respectively. In each plot, we illustrate the 2D spectrum in the top panel centering at the center of the stellar component of the blob, while the bottom panel shows 1D spectrum at the corresponding observed wavelengths. The centers of [O II] and $\mathrm{H} \beta$ lines are indicated with the dashed gray lines. The shade regions in some figures indicate the area with poor S/N ratios in the spectrum .

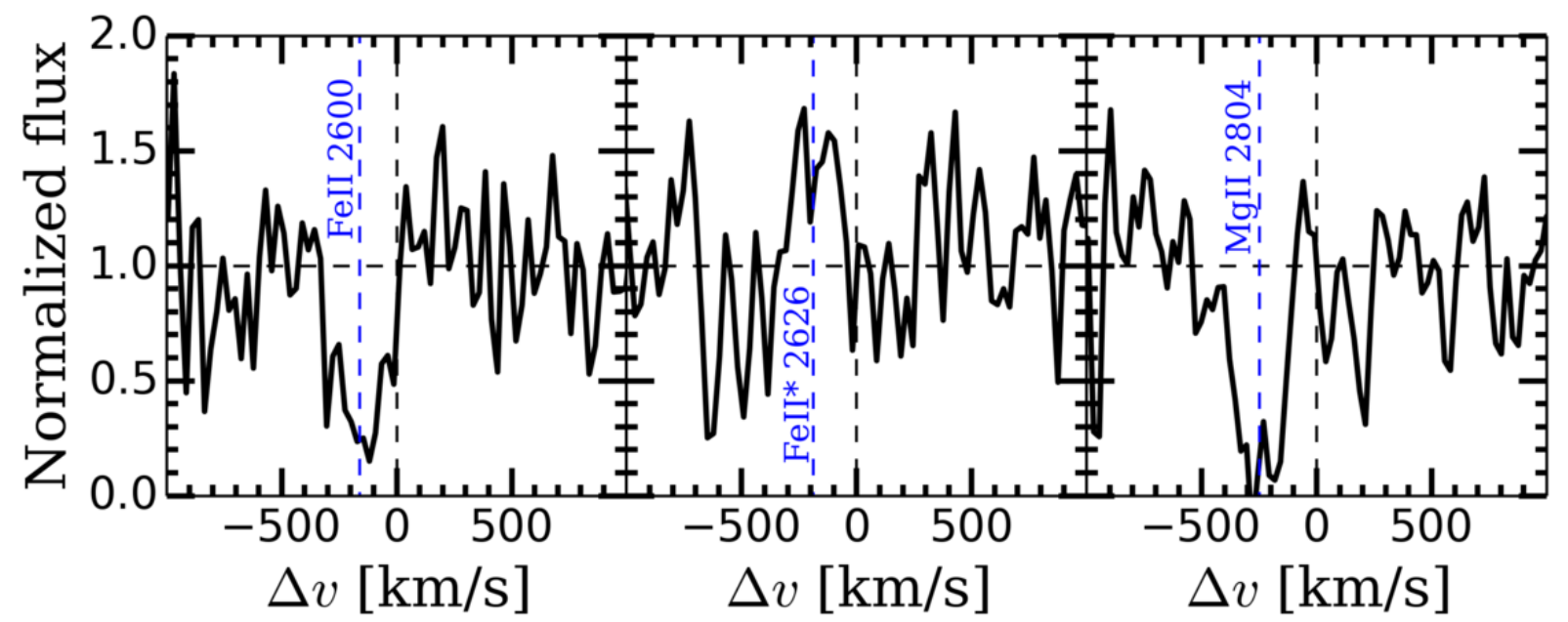

Figure 3. The blueshifted Fe II $\lambda 2600$ absorption (left), Fe II ${ }^{*} \lambda 2626$ fine-structure emission (middle), and Mg II $\lambda 2804$ absorption lines (right) of O IIIB-1. The observed flux is normalized to the continuum spectrum. The blue vertical dashed lines indicate the centroids of velocity offsets of each line. 
tion, Fe II ${ }^{*} \lambda 2626$ fine-structure emission, and $\operatorname{Mg}$ II $\lambda 2804$ absorption lines. They are all blueshifted from the systemic redshift, indicating the gas outflow from the galaxy. We fit the blueshifted absorption and emission lines with a single Gaussian profile. The velocity offsets of the Fe II, Fe II*, and $\mathrm{Mg}$ II lines are $-160 \mathrm{~km} \mathrm{~s}^{-1},-200 \mathrm{~km} \mathrm{~s}^{-1}$, and $-270 \mathrm{~km} \mathrm{~s}^{-1}$, respectively. It is important to note that the $\mathrm{Mg}$ II absorption line is possibly affected by strong resonant emission at the systemic velocity. This infilling emission can shift the centroid of the $\mathrm{Mg}$ II velocity offset in the order of tens $\mathrm{km} \mathrm{s}^{-1}$ (e.g., Prochaska et al. 2011). Erb et al. (2012) studied the outflow of normal star-forming galaxies at $z=1-2$ by using various interstellar absorption lines including those detected in this work. They found that the velocity offsets of the Fe II $\lambda 2600$ and $\mathrm{Mg}$ II $\lambda 2804$ absorption lines of star-forming galaxies at $z=1-2$ with the stellar masses and star formation rates comparable to those of O IIIB-1 range between $-190 \mathrm{~km} \mathrm{~s}^{-1}$ and $-130 \mathrm{~km} \mathrm{~s}^{-1}$. The Fe II ${ }^{*}$ and $\mathrm{Mg}$ II lines of O IIIB-1 show the velocity offsets slightly higher than those of the normal starforming galaxies.

\subsection{AGN Diagnostics}

An AGN is one of plausible energy sources providing hard ionizing photons that can cause the emission lines at large scale. Yuma et al. (2017) carried out a primary check for the AGN contribution in the [O III] blobs by cross-matching the X-ray and radio catalogs obtained by Ueda et al. (2008) and Simpson et al. (2012), respectively. They found no X-ray or radio counterpart for any [O III] blobs in our sample down to the X-ray luminosity of $10^{42} \mathrm{erg} \mathrm{s}^{-1}$ as mentioned in Section 2 This luminosity limit is $\sim 0.5$ dex brighter than the faintest bin of the X-ray luminosity functions (XLFs) of AGNs at similar redshifts (e.g., Fotopoulou et al. 2016, Ranalli et al. 2016). So we know that the [O III] blobs are not an unobscured AGN, but the possibility that the [O III] blobs are faint or heavily obscured AGNs cannot yet be ruled out.

To further investigate AGN signature in the targets, we first plot the Baldwin, Phillips, and Terlevich (BPT) digram in the left panel of Figure 4 The BPT diagram is a plot of the $[\mathrm{O} \mathrm{III}] / \mathrm{H} \beta$ ratio versus the $[\mathrm{N} \mathrm{II}] / \mathrm{H} \alpha$ ratio (Baldwin et al. 1981). Kewley et al. (2006) used this diagram to efficiently classify their sample into the star-forming galaxies, composites, and AGNs. Compared with star-forming galaxies, AGNs have the higher ionization state resulting in higher values of both $[\mathrm{O} \mathrm{III}] / \mathrm{H} \beta$ and $[\mathrm{N} \mathrm{II}] / \mathrm{H} \alpha$ ratios. We plot local galaxies $(z=0.04-0.10)$ obtained from the Sloan Digital Sky Survey (SDSS) Data Release 7 (DR7; Abazajian et al. 2009) in the left panel of the figure and separate them into pure starforming galaxies (red), composites (blue), and AGNs (green) based on their locations on the BPT diagram. O IIIB-1 is the only object that has the near infrared spectrum available. It is clearly seen from the figure that O IIIB-1 lies exactly on the distribution of the local star-forming galaxies indicating that it is a normal star-forming galaxy.

For the remaining three [O III] blobs, we use the blue diagram, which is a plot between the dust corrected $[\mathrm{O} \mathrm{III}] / \mathrm{H} \beta$ and $[\mathrm{O} \mathrm{II}] / \mathrm{H} \beta$ emission line ratios (the right panel of Figure 4). AGNs can be distinguished from the star-forming galaxies with the solid curve shown in the right panel of Figure 4 (e.g., Lamareille 2010; Harikane et al.2014). On the blue diagram, we display the local SDSS galaxies that we already categorized into star-forming galaxies, composites, and AGNs based on the BPT diagram as a reference. The local starforming galaxies and composites are largely overlapped with each other in the region below the curve that is used to separate the star formation activity from AGNs. On the other hand, AGNs can be distinguished from the star formation galaxies and composites efficiently. Lamareille (2010) stated that the contamination of AGN in the region of star-forming galaxies on the blue diagram is roughly $16 \%$. We also plot a Seyfert type-2 AGN (J2240-0927) at $z=0.326$ that shows the extended [O III] emission line (Schirmer et al. 2013). We discuss the similarity and difference between our [O III] blobs and this AGN in Section 4.7

From the blue digram in the right panel of Figure 4, O IIIB1 is still confirmed to be in the pure star formation region consistent with its location on the BPT diagram. O IIIB-2, on the other hand, shows the higher $[\mathrm{O} \mathrm{II}] / \mathrm{H} \beta$ ratio and get closer to the separation curve. Thus we cannot rule out the possibility that O IIIB-2 might be fueled by an AGN activity. The other two [O III] blobs at $z \sim 0.83$ show the remarkably high $[\mathrm{O} \mathrm{III}] / \mathrm{H} \beta$ ratios. O IIIB-3 is clearly located in the AGN region. This is in good agreement with its emission-line width mentioned in Section 4.2. The [O III] $\lambda 5007$ line width of $419.5 \mathrm{~km} \mathrm{~s}^{-1}$ suggests that O IIIB-3 is plausibly the type-2 Seyfert, whose typical line widths are roughly $\sim 500 \mathrm{~km} \mathrm{~s}^{-1}$.

$\mathrm{O}$ IIIB-4 shows the remarkably high $[\mathrm{O}$ III $] / \mathrm{H} \beta$ ratio but low $[\mathrm{O} I I] / \mathrm{H} \beta$ ratio (the right panel of Figure 4). The high ratio of [O III] to $\mathrm{H} \beta$ emission lines places O IIIB-4 above the separated curve in the blue diagram. As seen in the blue diagram, some fraction of the local star-forming galaxies distribute in the same region as O IIIB-4, while AGNs do not. It is likely that $\mathrm{O}$ IIIB-4 is one of the star-forming galaxies with the strongest $[\mathrm{O} \mathrm{III}] / \mathrm{H} \beta$ ratio. In conclusion, with the emission-line diagnostics, we can confirm that O IIIB-1 and O IIIB-4 are star-forming galaxies, whereas O IIIB-3 is an obscured AGN. However, there is no clear conclusion for O IIIB2 ; it can be a star-forming galaxy, composite galaxy, or AGN.

\subsection{Properties of ISM}

Nakajima et al. (2013) and Nakajima \& Ouchi (2014) show that the diagram between the [O III]/[O II] line ratio and the R23 index is efficient to investigate an ionization parameter and metallicity of a star-forming galaxy simultaneously. The emission-line ratio of an atom at different ionization states such as the $[\mathrm{O} \mathrm{III}] /[\mathrm{O} \mathrm{II}]$ ratio strongly depends on the ionization parameter of gas, $q_{i o n}$, which is the ratio of the ionizing photon flux per unit area to the number density of hydrogen atoms. The ionization parameter $q_{i o n}$ corresponds to the dimensionless ionization parameter $U$ as $U \equiv q_{i o n} / c$, where $c$ is the speed of light (Kewley \& Dopita 2002). The $R 23$ index is defined as

$$
R 23=\frac{[\mathrm{O} \mathrm{II}] \lambda 3727+[\mathrm{O} \mathrm{III}] \lambda \lambda 4959,5007}{\mathrm{H} \beta} .
$$

The $R 23$ index is one of the good indicators for estimating metallicities of the galaxies (e.g., Pagel et al. 1979, Kewley \& Dopita 2002), although it slightly depends on the ionization parameter.

We plot the $[\mathrm{O} \mathrm{III}] /[\mathrm{O} \mathrm{II}]$ ratios of the $[\mathrm{O} \mathrm{III}]$ blobs against their $R 23$ indices in Figure 5 to investigate the properties of their interstellar media (ISM). The local SDSS star-forming galaxies (gray dots), local SDSS AGNs (dark gray dots), and star-forming galaxies at $0.47<z<0.92$ (open triangles; Lilly et al. 2003) are also plotted as a reference. Another population that we plot in the Figure as a reference is Green Peas (hereafter GPs). GPs are the compact star-forming galaxies at 

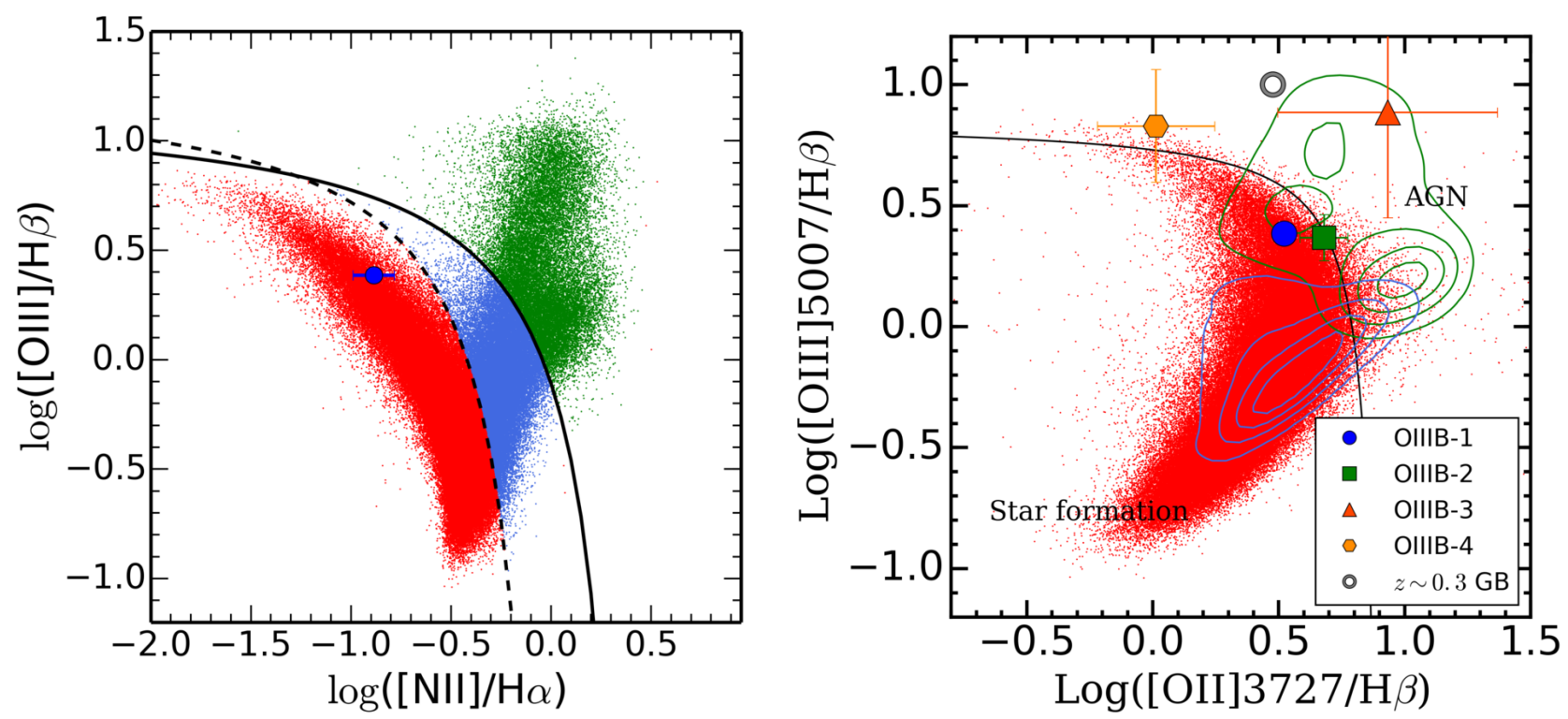

Figure 4. Left panel: A BPT diagram showing the $[\mathrm{O} \mathrm{III}] / \mathrm{H} \beta$ ratio against the $[\mathrm{N} \mathrm{II}] / \mathrm{H} \alpha$ ratio. The blue circle indicates $\mathrm{O}$ IIIB-1, while local galaxies taken from SDSS DR7 data are shown in three different colors. The red, blue, and green dots are for star-forming galaxies, composites, and AGNs, respectively. The black dashed and solid curves are the criteria for separating pure star-forming galaxies, composites, and AGNs by Kauffmann et al. (2003) and Kewley et al. (2001), respectively. Right panel: $[\mathrm{O} \mathrm{III}] / \mathrm{H} \beta$ versus $[\mathrm{O} \mathrm{II}] / \mathrm{H} \beta$ or the blue diagram of four $[\mathrm{O} \mathrm{III}]$ blobs at $z=0.63$ and $z=0.83$. The line ratios are already corrected for dust attenuation. The blue circle is O IIIB-1 and the green square is O IIIB-2 at $z \sim 0.63$. O IIIB-3 and O IIIB-4 at $z \sim 0.83$ are indicated with the red triangle and the orange hexagon, respectively. Red dots represent the local star-forming galaxies classified by the BPT diagram in the left panel. Blue and green contours show the distribution of composites and AGNs, respectively. The open gray circle shows a Seyfert type-2 AGN (J2240-0927) at $z \sim 0.3$ with spatially extended [O III] emission line, which is called a green bean (Schirmer et al. 2013). The black solid line divides the blue diagram into two regions of star formation and AGN (Lamareille 2010$)$.
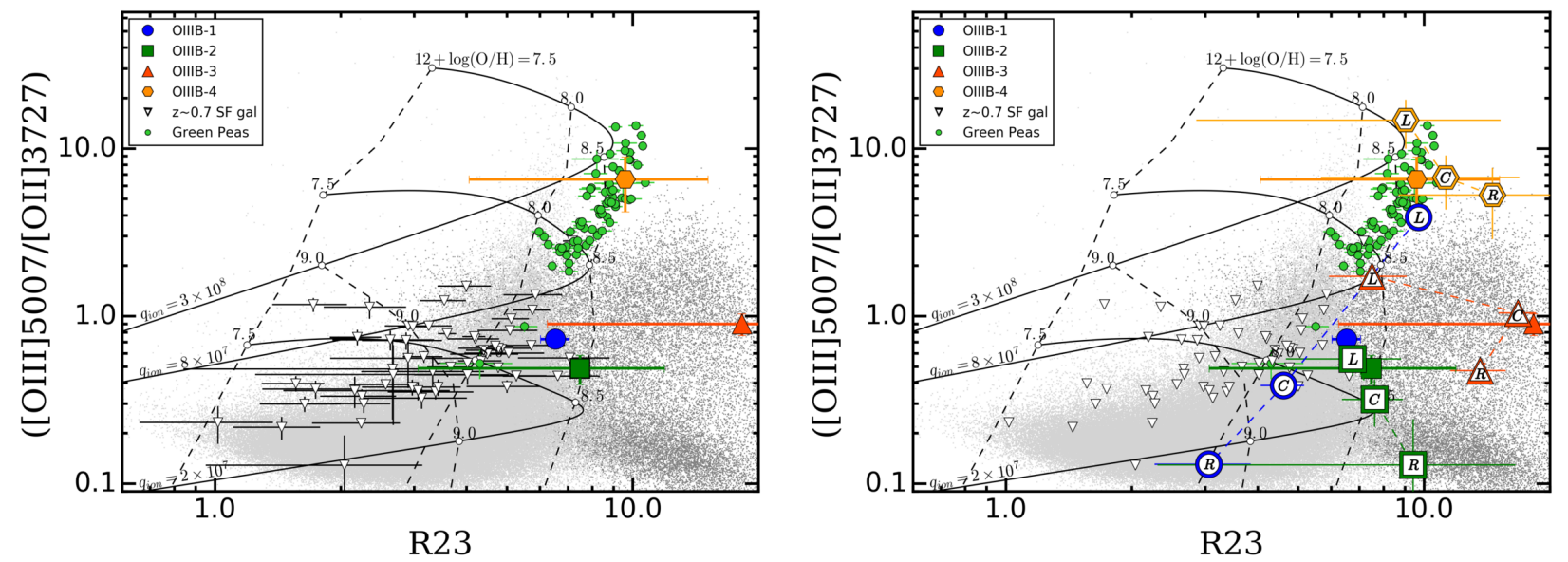

Figure 5. The relation between $[\mathrm{O} \mathrm{III}] /[\mathrm{O} \mathrm{II}]$ ratio and $R 23$ index. Left panel: Colored symbols represent $\mathrm{O}$ IIIBs at $z \sim 0.7$ as in previous figures. Gray and dark gray dots are the SDSS star-forming galaxies and AGNs, respectively. Open triangles represent star-forming galaxies at $z=0.5-0.9$ by Lilly et al. (2003). Green circles are for the local green peas with the strong [O III] emission line by Cardamone et al. (2009). Each solid black line shows a track of photoionization models at a constant ionization parameters of $q_{i o n}=2 \times 10^{7}, 8 \times 10^{7}$, and $3 \times 10^{8} \mathrm{~cm} \mathrm{~s}^{-1}$ with $12+\log (\mathrm{O} / \mathrm{H})$ in the ranges of $7.5-9.0$ (Kewley \& Dopita 2002). The dashed lines connect the photoionization model tracks at a constant $12+\log (\mathrm{O} / \mathrm{H})$. Right panel: $[\mathrm{O} \mathrm{III}] /[\mathrm{O} \mathrm{II}]$ ratio vs $R 23$ index of the radial profiles of the [O III] blobs. Opened symbols with the letters "L", "C", and "R" refer to the left, central, and right parts of the blobs in Figure 6 (More details in context). Other symbols and lines are identical to those in the left panel.

$z=0.112-0.360$ with the strong [O III] emission line with the rest-frame equivalent width up to $1,000 \AA$ (Cardamone et al. 2009). The [O III] emission line of GPs falling into the $r$ band makes this type of galaxies looks green in the $g, r, i$ composite image. In addition to the observed galaxies at various redshifts, we plot photoionization models at constant ionization parameters of $q_{i o n}=2 \times 10^{7}, 8 \times 10^{7}$, and $3 \times 10^{8} \mathrm{~cm} \mathrm{~s}^{-1}$ with varying metallicities. The models with the constant metallicities are linked with dashed lines and the values of the metallicities in terms of $12+\log (\mathrm{O} / \mathrm{H})$ are indicated by the open cir- cles. The local SDSS star-forming galaxies show a variety of $R 23$ indices ranging from 1.0 to 10.0 , while their [O III]/[O II] ratios are mostly below 1.0. Likewise, star-forming galaxies at $z \sim 0.7$ are distributed in the same area on the plot. It is indicated that most of the star-forming galaxies at $z=0$ and $z \sim 0.7$ have ionization parameters between $q_{\text {ion }}=2 \times 10^{7}$ $\mathrm{cm} \mathrm{s}^{-1}$ and $q_{\text {ion }}=8 \times 10^{7} \mathrm{~cm} \mathrm{~s}^{-1}$ but have wide ranges of metallicities. On the other hand, the star-forming GPs with the strong [O III] emission line show the high [O III]/[O II] ratios and the R23 indices. According to the figure, the GPs 


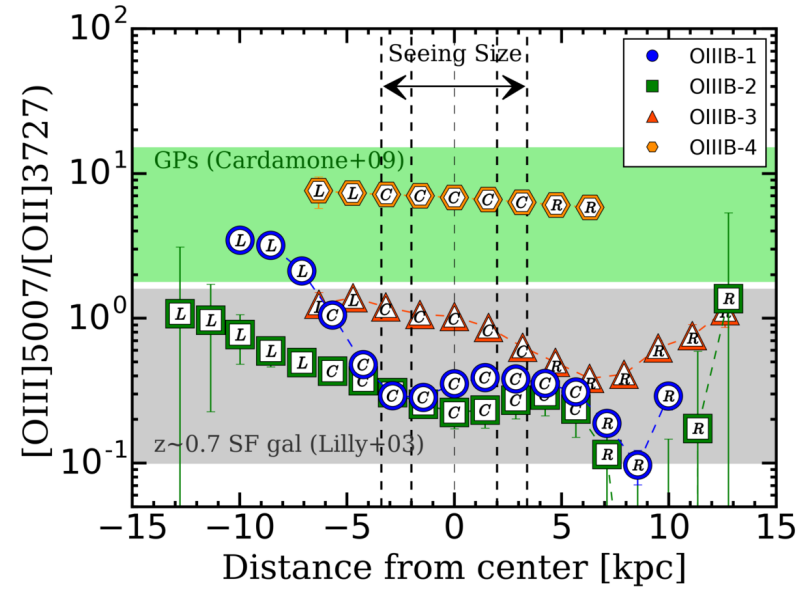

Figure 6. Radial profiles of $[\mathrm{O} \mathrm{III}] /[\mathrm{O} \mathrm{II}]$ ratios of $4 \mathrm{O}$ IIIBs at $z \sim 0.7$ as a function of the distance from the center of the blobs. The left-to-right radial profile is drawn from the top to the bottom part of the 2D spectrum in Figure 2 The symbols of 4 [O III] blobs are identical to the previous figures. The letters "L", "C", and "R" indicate the regions that we use to determine the left, central, and right components of a given blob. The grey area represents the typical line ratios of star-forming galaxies at $0.47<z<0.92$ (Lilly et al. 2003). The green region indicates the line ratios of the green peas at $z=$ $0.112-0.360$ by Cardamone et al. (2009). The average seeing size of the data is also shown in the figure.

at $z=0.1-0.4$ show the ionization parameters higher than $8 \times 10^{7} \mathrm{~cm} \mathrm{~s}^{-1}$, which is clearly higher than the normal starforming galaxies at $z=0$ and $z=0.7$. Due to the strong [O II] and [O III] emission, the SDSS AGNs at $z \sim 0$ show high $R 23$ indices that is relatively greater than those of the star-forming galaxies at $z=0$ and $z \sim 0.7$. Meanwhile, the [O III]/[O II] line ratios of the local AGNs are distributed in the same range as those of the star-forming galaxies.

O IIIB-1 and OIIIB-2 are located in the same region as the local and $z=0.7$ star-forming galaxies suggesting similar ionization parameters and metallicities. O IIIB-1 and O IIIB-2 should have the ionization parameter slightly less than $q_{i o n}=8 \times 10^{7} \mathrm{~cm} \mathrm{~s}^{-1}$. Note that the inferred ionization parameter and metallicies of O IIIB-2 only apply if it is a starforming galaxy. O IIIB-3 show huge error bars in the $R 23$ index due to the low $\mathrm{S} / \mathrm{N}$ ratio of the $\mathrm{H} \beta$ emission line. As O IIIB-3 is classified as an AGN in Section 4.4 it is obvious that its location is consistent with the AGNs found in the local universe. In contrast, O IIIB-4 is located in the totally different region in Figure 5. O IIIB-4 show the impressively high [O III $] /[\mathrm{O}$ II $]$ ratio indicating that the ionization parameter $q_{\text {ion }}$ should be roughly $3 \times 10^{8} \mathrm{~cm} \mathrm{~s}^{-1}$. This high [O III]/[O II] ratio of O IIIB-4 agrees well with those of the GPs at $z=0.1-0.4$.

\subsection{Radial Profile of the [O III] blobs}

We further investigate the nature of the [O III] blobs by plotting their $[\mathrm{O} \mathrm{III}] /[\mathrm{O} \mathrm{II}]$ line ratios as a function of a distance from the center of the blobs. By observing the [O III] blobs with the slits finely aligned along the most extended direc-

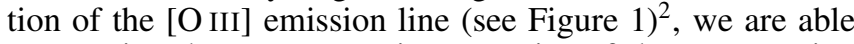
to examine the spectroscopic properties of the outer region of the [O III] blobs. The radial profile of the emission line is constructed from the background-subtracted 2D spectrum by integrating the emission line at each spatial position along the wavelength direction covering the full wavelength range of

\footnotetext{
2 This is not the case for O IIIB-2 and O IIIB-4, whose slit direction is fixed to that of $\mathrm{O}$ IIIB-3, the brightest [O III] blob in the same mask (see Section 3.1 .
}

the best-fitted Gaussian profile. This wavelength range covers all spatial components of the emission line. The stellar continuum profiles at the wavelengths blueward and redward of each emission line for a given blob are estimated by creating the continuum profiles using the identical wavelength range as used to create the emission-line profile. Then we interpolate both continuum profiles to obtain the average continuum profile at the wavelength of the emission line. In this way, we can ensure that the emission-line profile is not over- or under-estimated. The spatial profile of the $[\mathrm{O} \mathrm{III}] /[\mathrm{O} \mathrm{II}]$ ratio is obtained after carefully removing the estimated stellar continuum and shown in Figure 6. The negative values of the distance from the center refer to the component at the top of 2D spectral image in Figure 2 For comparison purpose, we show the shaded regions where the GPs at $z=0.1-0.4$ and star-forming galaxies at $z \sim 0.7$ are typically located.

It is seen from Figure 6 that all of our four samples extend beyond the seeing size of the spectral images. The center of O IIIB-1, O IIIB-2, and O IIIB-3 lie in the region where the typical star-forming galaxies at $z \sim 0.7$ are located. Interestingly, the left part of the O IIIB-1 profile at the distance larger than $5 \mathrm{kpc}$ show the high $[\mathrm{O} \mathrm{III}] /[\mathrm{O} \mathrm{II}]$ ratio consistent with the GPs at $z-0.1-0.4$. Similarly, the $[\mathrm{O}$ III $] /[\mathrm{O}$ II $]$ ratio of O IIIB-2 increases as a function of the distance from the center on both sides, albeit the large uncertainty. O IIIB-3 shows the $[\mathrm{O} \mathrm{III}] /[\mathrm{O} \mathrm{II}]$ ratio of roughly $1-2$, which is slightly higher than the central parts of O IIIB-1 and O IIIB-2, but it is still in the ranges of the typical star-forming galaxies. Finally, the $[\mathrm{O} \mathrm{III}] /[\mathrm{O} \mathrm{II}]$ radial profile of $\mathrm{O} \mathrm{IIIB}-4$ seems to be almost constant at roughly $[\mathrm{O} \mathrm{III}] /[\mathrm{O} \mathrm{II}]=5-10$ over $\sim 14 \mathrm{kpc}$. It is noteworthy that the spatial extension of O IIIB-4 is smaller than the other three blobs because the slit direction is unfortunately not designed to observe the longest axis of O IIIB-4. The $[\mathrm{O} \mathrm{III}] /[\mathrm{O} \mathrm{II}]$ ratios are significantly greater than those of the typical star-forming galaxies and consistent with those of the compact GPs at $z-0.1-0.4$. This is in agreement with what we have found in Section 4.5 (the left panel of Figure 5) that O IIIB-4 have both [O III]/[O II] ratio and $R 23$ index comparable to those of the GPs. In contrast to O IIIB-4, the GPs show compact morphology in $g, r, i$ images suggesting that they have a compact stellar component Cardamone et al. (2009). Although there is no detailed study about the extension of the [O III] emission line of the GPs yet, the compact size of the GPs seen in the $r$-band image, in which the [O III] emission line falls, may imply the compact size of the [O III] emission line as well. The unresolved morphology of the GPs in in the $r$-band image suggested that the sizes of the [O III] emission line should be less than $5 \mathrm{kpc}$ (Cardamone et al. 2009). Further study of spatial extension of the [O III] emission line for the GPs is necessary to confirm the above statement. The fact that O IIIB-4 show an extended profile of the [O III] emission line over $\sim 14 \mathrm{kpc}$ makes O IIIB-4 different in sizes from the GPs at $z=0.1-0.4$. So we call O IIIB-4 a giant green pea as it shows the high $[\mathrm{O} \mathrm{III}] /[\mathrm{O} \mathrm{II}]$ ratio like the GPs and its spatially extended feature of the [O III] emission line.

We replot the diagram of the $[\mathrm{O} \mathrm{III}] /[\mathrm{O}$ II $]$ ratio against the $R 23$ index in the right panel of Figure 5 by separating the radial profiles of each [O III] blob into 3 components: the central part and two outer regions on both sides of the profile beyond the stellar continuum. We use letters " $L$ ", " $C$ ", and " $\mathrm{R}$ " to represent the left, central, and right components of each blob, respectively. The left component corresponds to the left part of the radial profile shown in Figure 6 . The cen- 
tral component is roughly twice the FWHM of the continuum radial profile to ensure that the extended parts are not contaminated by the stellar contribution. As seen in the figure, the left and central components of O IIIB-2 are close to the plot of the entire object and are located in the region where the star-forming galaxies at $z \sim 0$ and $z \sim 0.7$ are distributed. The line ratio of the right sub-region of O IIIB-2, however, shows higher $R 23$ index and agrees well with the line ratios of the local SDSS AGNs. Originally, O IIIB-3 shows the high $R 23$ index with the large uncertainty. When we divide $\mathrm{O}$ IIIB3 into three components, the extended parts of O IIIB-3 show slightly lower $R 23$ indices, but are still consistent within the large uncertainties. The left part of O IIIB-3 falls closer to the GP region with the $[\mathrm{O} \mathrm{III}] /[\mathrm{O} \mathrm{II}]$ ratio higher than that of the central part, which is in agreement of Figure 6. Meanwhile, the central and right sub-regions are located close to the integrated line ratio.

For O IIIB-1, the left component moves apart from the rest and falls into the GP region of the high [O III]/[O II] ratios and $R 23$ indices. It can be argued that the extended component of O IIIB-1 does not have only the comparable [O III]/[O II] line ratio to the GPs as seen in Figure 6, but it also have the similar $R 23$ index. Likewise, the locations of all three components of $\mathrm{O}$ IIIB-4 on the $[\mathrm{O} \mathrm{III}] /[\mathrm{O} \mathrm{II}]-R 23$ diagram are consistent with those of the GPs. In conclusion, one extended part of O IIIB-1 and the entire O IIIB-4 show the [O III]/[O II] ratios and the $R 23$ indices consistent with those of the compact GPs at $z=0.1-0.4$.

\subsection{Discovery of A Giant Green Pea at $z=0.838$}

We discover a giant GP, O IIIB-4, which has spatially extended [O III] emission and line ratios consistent with those of the GPs found at $z=0.1-0.4$ by Cardamone et al. (2009). Figure 7 shows the close-up RGB image of O IIIB-4. The extended green color in the order of $10-20 \mathrm{kpc}$ around the center of O IIIB-4 clearly indicates the large-scale extension of the $[\mathrm{O} \mathrm{III}]$ emission line. It is noteworthy that the green components in the North-East (top left) direction of O IIIB-4 are also parts of O IIIB-4 when we measure the [O III] surface brightness down to $1.2 \times 10^{-18} \mathrm{erg} \mathrm{s}^{-1} \mathrm{~cm}^{-2} \operatorname{arcsec}^{-2}$ (cf. Figure 1).

The left panel of Figure 8 shows the relation between the rest-frame [O III] equivalent widths of the [O III] blobs and the SFRs derived by using the SED fitting method. In Yuma et al. (2017), the observed SEDs of the [O III] blobs are collected from the photometry in 9 bands: $B V R i z J H K$, IRAC ch1 $(3.6 \mu \mathrm{m})$, and IRAC ch2 $(4.5 \mu \mathrm{m})^{3}$. The magnitudes in the $i$ or $z$ band are excluded because they might be contaminated by the strong [O III] emission line at $z=0.63$ or $z=0.83$, respectively. The stellar population models are constructed with the Bruzual \& Charlot (2003) code by assuming the constant star formation history, the Salpeter (1955) initial mass function with the mass cutoffs of 0.1 and $100 M_{\odot}$, Calzetti et al. (2000) dust attenuation, and the solar metallicity. The effect of varying the parameters used to construct the models (e.g., the IMF, star formation histories, and metallicity) is included in the uncertainties of the derived stellar properties. The rest-frame [O III] EWs of all [O III] blobs are in the ranges of 200-1000 $\AA$. The giant green pea (O IIIB-4) shows the largest EW of $845 \pm 27 \AA$. We also plot the GPs at $z=0.1-0.4$ in the figure

\footnotetext{
${ }^{3}$ IRAC ch1 and ch2 are the mid-infrared data obtained with the Infrared Array Camera from the Spitzer UKIDSS Ultra Deep Survey (SpUDS; PI: J. Dunlop)
}

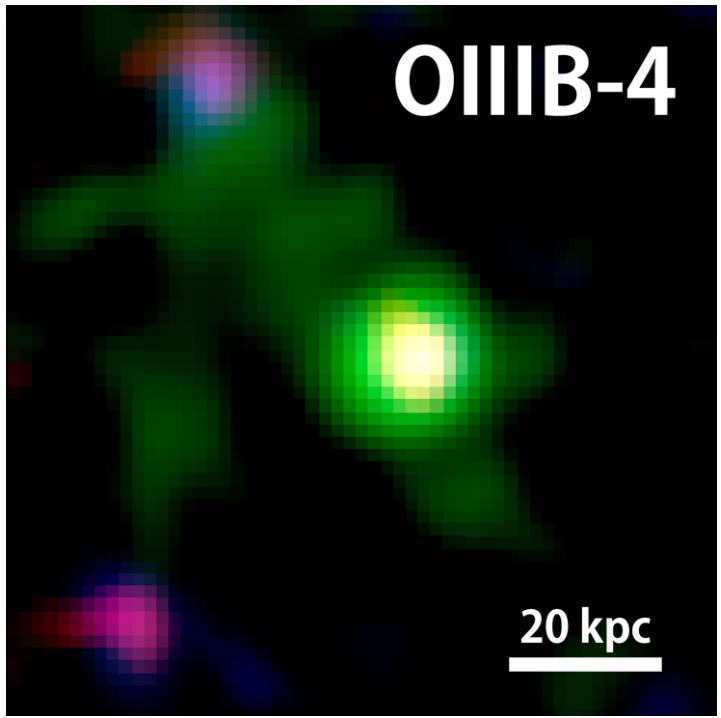

Figure 7. The composite images of O IIIB-4. North is up; East is left. The red and blue colors of both images are the images observed in the $R$ and $z$ bands, respectively, whereas the green color represents the [O III] emission line observed in the NB921 image. The size of each image is roughly $12 \times$ $12 \operatorname{arcsec}^{2}$, which correspond to the physical dimension of $90 \times 90 \mathrm{kpc}^{2}$ at $z=0.8379$. The white bar at the bottom right corner indicates the physical scale of $20 \mathrm{kpc}$.

for comparison purpose. As mentioned earlier, the GPs are selected as the galaxies with excessive fluxes in the $r$-band image. So they should show very high rest-frame [O III] EWs by definition. The SFRs of the GPs at $z=0.1-0.4$ were derived from the dust-corrected $\mathrm{H} \alpha$ fluxes (Cardamone et al. 2009). From the left panel of Figure 8, the rest-frame [O III] EWs of the GPs are typically in the ranges of $100-2000 \AA$. All [O III] blobs studied in this paper show the EWs in well agreement with the GPs at $z=0.1-0.4$. However, O IIIB-1 and O IIIB-2 have significantly higher SFRs than the GPs, indicating more intense activity of star formation. Meanwhile, the giant green pea (O IIIB-4) show the lower SFR of roughly $15 M_{\odot} \mathrm{yr}^{-1}$, which is consistent with the compact GPs at $z=0.1-0.4$.

We plot the radial profile of the rest-frame [O III] EW in the right panel of Figure 8 by dividing the profile into 3 components based on their distances from the center: left $(L)$, center $(C)$, and right $(R)$. O IIIB-4 shows high [O III] EWs in all components. The left and central parts of O IIIB-4 have the [O III] EW in the ranges of $800-1000 \AA$, consistent with the EW of the entire blob. They are in the same ranges as the typical GPs at $z=0.1-0.4$. The right component even shows the higher EW, albeit the large error bar due to the low $\mathrm{S} / \mathrm{N}$ ratio of the faint continuum. This clearly confirms that O IIIB-4 have the [O III] EW high enough to be call a giant green pea. Among the remaining 3 [O III] blobs, O IIIB-3 shows the high [O III] EW comparable to the GPs throughout the entire object. On the other hand, O IIIB-1 and O IIIB-2 show the [O III] EWs at the center slightly smaller than those of the typical GPs at $z=0.1-0.4$. Their [O III] EWs are in the order of 100 $\AA$, while the [O III] EWs of the extended components increase to approximately $150-250 \AA$ in the case of O IIIB-2. The left component of O IIIB-1 shows the significantly large EW of more than $1000 \AA$. It is consistent with what we have found in the right panel of Figure 5 that the left part of O IIIB-1 have the $[\mathrm{O} \mathrm{III}] /[\mathrm{O} \mathrm{II}]$ line ratio and the $R 23$ index comparable to those of the GPs. 

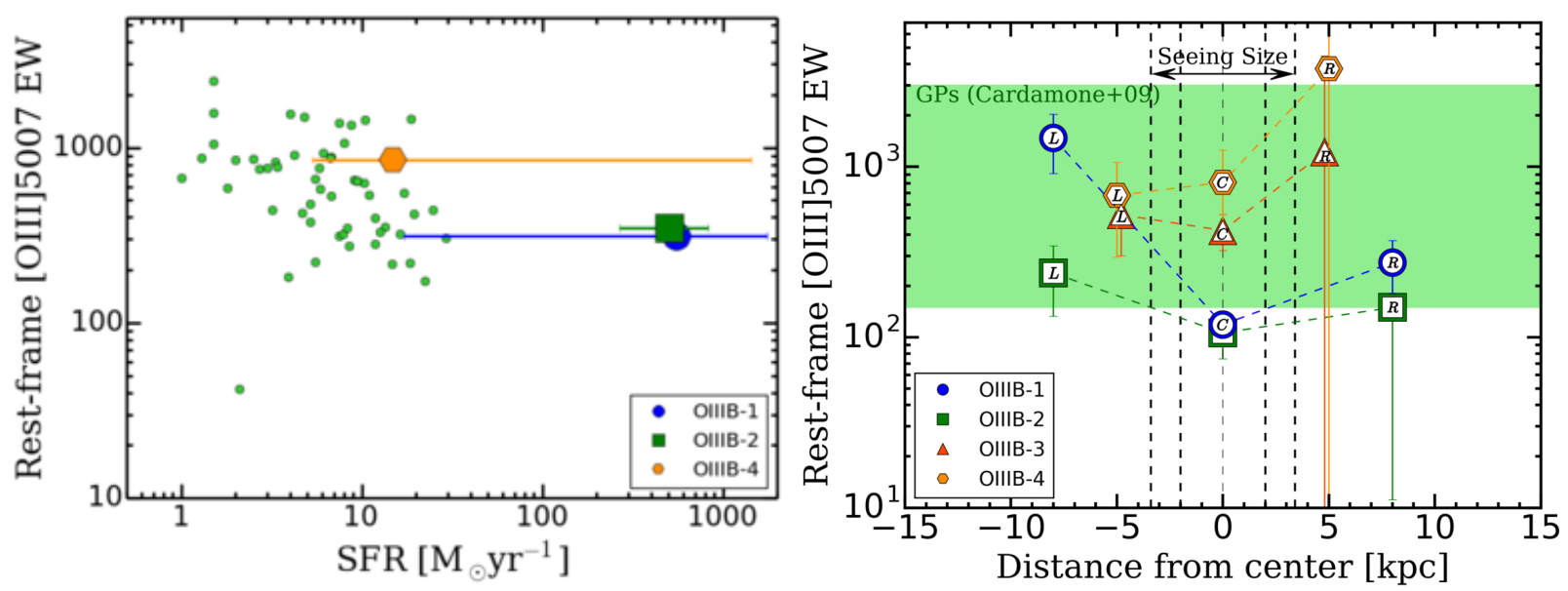

Figure 8. Left panel: A relation between the rest-frame [O III] equivalent widths ([O III] EWs) and the star formation rates (SFRs) of 4 [O III] blobs at $z \sim 0.63$ and $z \sim 0.83$. The symbols of the [O III] blobs are identical to those in the previous figures. The small green circles show the green peas discovered at $z=0.1-0.4$ by Cardamone et al. (2009). Right panel: The rest-frame [O III] EWs of the [O III] blobs as a function of the distance from the center. The radial profile of each [O III] blob is divided into 3 components: left (L), center (C), and right (R), corresponding to the left, center and right parts of the profile shown in Figure 6

Besides the GPs at $z=0.1-0.4$, there is another population that is similar to our giant green pea. Schirmer et al. (2013) discovered Seyfert-2 galaxies at $z \sim 0.3$ in SDSS DR8 with luminous narrow-line regions and named these galaxies green beans (GBs). GBs show the high [O III] luminosities in the order of $10^{43} \mathrm{erg} \mathrm{s}^{-1}$ and large [O III]/[O II] ratios of more than 1.0. Compared with GPs at similar redshifts, GBs are much larger with the size of the [O III] emission line of $15-20 \mathrm{kpc}$ and show higher $[\mathrm{O} \mathrm{III}] /[\mathrm{O} \mathrm{II}]$ ratios. Their $[\mathrm{O} \mathrm{III}]$ EWs are in the order of $1000 \AA$. GBs at $z \sim 0.3$ are similar to O IIIB4 in terms of the large [O III] EW and the spatial extension of the [O III] emission line. However, the [O III $] /[\mathrm{O}$ II $]$ ratios of GBs are slightly higher than those of O IIIB-4. The main difference between O IIIB-4 and GBs is the energy source; GBs are Seyfert type-2 AGNs but O IIIB-4 is a star-forming galaxy. We plot the most prominent GB, J2240-0927, in the right panel of Figure 4. Its location on the blue diagram is consistent with the distribution of local SDSS AGNs, whereas O IIIB-4 is located close to the local star-forming galaxies on the same figure. Additionally, the high-ionization lines such as $[\mathrm{Ne} \mathrm{V}]$ emission are not detected in O IIIB-4. The $3 \sigma$ flux limit for non-detection is roughly $1.0 \times 10^{-18} \mathrm{erg} \mathrm{s}^{-1} \mathrm{~cm}^{-2}$, which provides the $3 \sigma$ upper limit on the $[\mathrm{Ne} \mathrm{V}] / \mathrm{H} \beta$ ratio of $\mathrm{O}$ IIIB-4 to be $<0.03$. In contrast, the $[\mathrm{Ne} \mathrm{V}]$ emission line is detected in many of GBs including J2240-0927 with the $[\mathrm{Ne} \mathrm{V}] / \mathrm{H} \beta$ ratio of 0.22 (Schirmer et al. 2016).

Figure 9 shows the correlation between the [O III]/[O II] line ratios and four different physical properties: the stellar mass $\left(M_{*}\right)$, star formation rate (SFR), specific SFR $\left(\mathrm{sSFR}=\mathrm{SFR} / M_{*}\right)$, and $\mu_{0.32}{ }^{4}$ The stellar mass and SFR of the [O III] blobs are derived by the SED fitting method (Yuma et al. 2017). $\mu_{0.32}$ is defined as a combination of $M_{*}$ and the SFR proposed by Mannucci et al. (2010):

$$
\mu_{\alpha}=\log _{10}\left(M_{*}\right)-\alpha \log _{10}(\mathrm{SFR})
$$

where $\alpha=0.32$ provides the minimum scatter of the SDSS galaxies in the local universe in the $\mu_{\alpha}$-metallicity plane. The metallicity in terms of $12+\log (\mathrm{O} / \mathrm{H})$ can be empirically esti-

\footnotetext{
${ }^{4}$ Note that we do not plot O IIIB-3 in Figure 9 because the AGN contribution might affect the SED fitting results.
}

mated from $\mu_{0.32}$ as

$$
12+\log (\mathrm{O} / \mathrm{H})=8.90+0.39 x-0.20 x^{2}-0.077 x^{3}+0.064 x^{4},
$$

where $x=\mu_{0.32}-10$ (Mannucci et al.2010). It is noteworthy that Equation 3 is obtained by fitting the local SDSS galaxies with stellar masses of $\log \left(M_{*}\right)=9-11 M_{\odot}$ or $\mu_{0.32}$ in the range of $\mu_{0.32}=9.0-11.5$. In this fitting range, the metallicity $12+\log (\mathrm{O} / \mathrm{H})$ decreases with $\mu_{0.32}$. We would reach the minimum metallicity of $12+\log (\mathrm{O} / \mathrm{H})=8.4$ at $\mu_{0.32}=8.73$. This relation might not be highly accurate for galaxies with $\mu_{0.32}$ smaller than 8.73. However, it seems that the metallicity continues to decrease at $\mu_{0.32}$ below 8.73 , but becomes constant at $\mu_{0.32}$ greater than 10.5. Mannucci et al. (2010) also proposed that the metallicity of star-forming galaxies with any stellar mass, SFR, and at any redshift up to $z=2.5$ practically follows the linear correlation between $12+\log (\mathrm{O} / \mathrm{H})$ and $\mu_{0.32}$ :

$$
12+\log (\mathrm{O} / \mathrm{H})=8.90+0.47 x \quad \text { if } \mu_{0.32}<10.2,
$$

where $x=\mu_{0.32}-10$. Therefore, $\mu_{0.32}$ can basically represent the metallicity of the galaxies with the stellar mass in the range of $\log \left(M_{*}\right)=9-11 M_{\odot}$.

As mentioned earlier, the giant GP, O IIIB-4, shows the highest $[\mathrm{O} \mathrm{III}] /[\mathrm{O} \mathrm{II}]$ ratio among all four [O III] blobs discussed in this paper. It is located in different locations from the rest of [O III] blobs in all plots in Figure 9] The stellar mass of O IIIB-4 is $6.75_{-2.91}^{+11.22} \times 10^{7} M_{\odot}$. By compared with the other [O III] blobs in our sample, O IIIB-4 is the [O III] blobs with the smallest $M_{*}$ and the lowest $\mu_{0.32}$. The SSFR of O IIIB-4 is $\sim 2 \times 10^{2} \mathrm{Gyr}^{-1}$, which is comparable to the other [O III] blobs. In fact, most of the [O III] blobs found at $z=0.63$ and $z=0.83$ show the high SSFR that is greater than the sSFR of typical star-forming galaxies on the so-called main sequence of the stellar mass-SFR diagram (Elbaz et al. 2007, Yuma et al. 2017). O IIIB-4 is the [O III] blob at $z \sim 0.83$ with the smallest stellar mass. Even if we compare O IIIB-4 with the normal [O III] emitters at the same redshift, we would still find that OIIB-4 is among the emitters with the smallest stellar mass and highest sSFR (cf. Figures 12 and 13 in Yuma et al. 2017). The high [O III]/[O II] line ratio of O IIIB-4 is comparable to the $z=0.1-0.4$ GPs; however, 

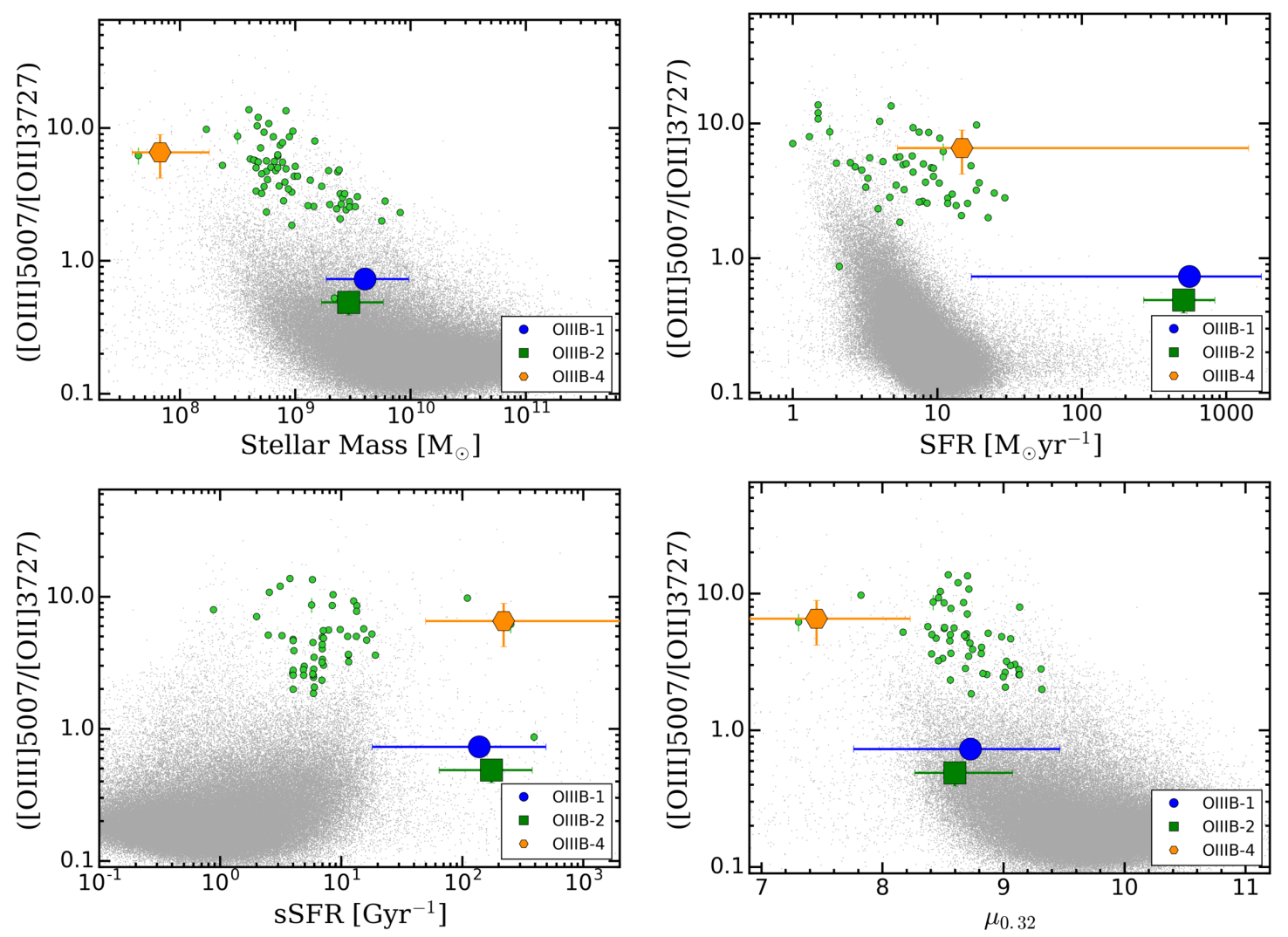

Figure 9. Diagrams of the $[\mathrm{O} \mathrm{III}] /[\mathrm{O} \mathrm{II}]$ ratios versus the stellar mass $\left(M_{*} ;\right.$ top left), star formation rate (SFR; top right), specific star formation rate (sSFR; bottom left), and $\mu_{0.32}$ (defined by Mannucci et al. (2010); bottom right). Symbols are identical to those in the previous figures.

their stellar properties are not exactly consistent. O IIIB-4 has the stellar mass smaller than a majority of the GPs and SDSS galaxies. The sSFR of O IIIB-4 is consistent with two GPs at $z=0.1-0.4$, while it is higher than those of the remaining GPs. The low $\mu_{0.32}$ of O IIIB-4 as seen in the bottom right panel of Figure 9 may imply its low metallicity. Amorín et al. (2010) showed that the GPs at $z=0.1-0.4$ have the metallicities in the ranges of $7.6<12+\log (\mathrm{O} / \mathrm{H})<8.4$. The comparable line ratios between O IIIB-4 and the GPs may imply that the metallicity of O IIIB-4 is likely similar to that of the GPs. The implied sub-solar metallicity of O IIIB-4 might affect the stellar properties derived by the SED fitting method in that the models with sub-solar metallicity give slightly lower SFRs $(0.12 \mathrm{dex})$ than those with solar metallicity (Yabe et al. 2009). However, the effect of varying metallicity of models is already included in the uncertainties of the derived properties.

With the small stellar mass and low metallicity, O IIIB-4 is somehow similar to the galaxies with extreme emission lines at low redshifts (e.g., Amorín et al. 2014; Izotov et al. 2017, Yang et al. 2017). Amorín et al. (2014) found the galaxies at $0.2 \leq z \leq 0.9$ with high rest-frame [O III] EWs of $100-1700 \AA$. They also suggested the high ionization parameters of $q \geq 10^{8} \mathrm{~cm} \mathrm{~s}^{-1}$, the sSFR of $1-100 \mathrm{Gyr}^{-1}$, and the metallicity of $7.5<12+\log (\mathrm{O} / \mathrm{H})<8.3$, which are similar to O IIIB-4. Amorín et al. (2014) also determined the $\mu_{0.32}$ of their sample to be in the range of $\mu_{0.32}=7.0-8.5$. This is also consistent with the $\mu_{0.32}$ of O IIIB-4. Izotov et al. (2017) and Yang et al. (2017) conducted separate surveys and found the galaxies with extreme $[\mathrm{O} \mathrm{III}] /[\mathrm{O} \mathrm{II}]$ emission-line ratios at $z<0.07$ and $z<0.05$, respectively. Yang et al. (2017) proposed the photometric method to select these galax- ies and called them the blueberry galaxies, which are basically GPs with low masses. The blueberry galaxies show low stellar masses and high [O III]/[O II] ratios comparable to those of O IIIB-4. However, the only obvious difference between $\mathrm{O}$ IIIB-4 and these extreme emission-line galaxies is the physical size of the emission line. These galaxies show the size so compact that some of them are unresolved in the SDSS images (Yang et al. 2017).

The average properties of O IIIB-1 are indistinguishable from those of O IIIB-2; it is located close to O IIIB-2 in all plots in Figure 9 Although the $\left[\mathrm{O}_{\mathrm{II}}\right] /[\mathrm{O} \mathrm{II}]$ line ratios of $\mathrm{O}$ IIIB-1and O IIIB-2 are not as high as those of O IIIB-4 and GPs, they are still somewhat higher than the majority of the local star-forming galaxies. According to the figure, O IIIB-1and O IIIB-2 show the moderate masses of roughly $3-5 \times 10^{9} M_{\odot}$, the significantly high SFR in the order of few hundreds $M_{\odot} \mathrm{yr}^{-1}$, and subsequently the high sSFR as compared with the local star-forming galaxies. Their $\mu_{0.32}$ values are less than most star-forming galaxies in the local universe, implying the metallicities lower than those of the local starforming galaxies.

\section{DISCUSSION}

We know from Section 4.4 that an AGN activity is possibly responsible for the spatial extension of the [O III] emission line in only one blob, O IIIB-3, but we cannot make a constraint on AGN contribution of O IIIB-2. O IIIB-2 can be a star-forming galaxy, composite, or AGN. So we do not further discuss about the origins of O IIIB-2 and O IIIB-3 because of the possible AGN contribution. The giant GP (O IIIB-4) with the remarkably high $[\mathrm{O} \mathrm{III}] /[\mathrm{O} \mathrm{II}]$ line ratios and $\mathrm{O}$ IIIB-1 are 
likely to be heated by the star formation activity with the high sSFR. Jaskot \& Oey (2013) studied six most extreme GPs at $z=0.1-0.4$ with the highest $[\mathrm{O}$ III]/[O II] ratios. They examined possible origins of the He II $\lambda 4686$ emission detected in most of their sample including Wolf-Rayet stars, AGNs, and fast radiative shocks. Because of the size difference of the [O III] emission lines between the GPs at $z=0.1-0.4$ and [O III] blobs at $z \sim 0.7$, the physical structures in the extended regions of the [O III] blobs may be different from the GPs. In this section, we discuss plausible scenarios and physical conditions that maybe able to explain the extended [O III] emission in O IIIB-1 and O IIIB-4 including an AGN-light echo (e.g. Schawinski et al. 2015; Ichikawa et al.2018), fast radiative shocks (e.g. Kewley et al. 2001; Allen et al. 2008; Shirazi \& Brinchmann 2012), and the density bounded system (e.g. Nakajima et al. 2013, Nakajima \& Ouchi 2014).

\subsection{AGN-Light Echo}

An AGN plays an important role in regulating the galaxy evolution. The accretion to the supermassive black hole at the center of the AGN produces the significant amount of radiative energy that is able to photoionize the interstellar medium of the host galaxy and cause large-scale gas outflows from the host galaxy (e.g., Silk \& Rees 1998). In this mode of the AGN activity, AGN photoionization overcomes the photoionization from the H II region of the host galaxy, so the host galaxy can be identified as an AGN (e.g., Baldwin et al. 1981; Veilleux \& Osterbrock 1987, Lamareille 2010). It has been long known that an AGN can show luminosity variability (Ulrich et al. 1997). It is possible that the AGN photoionization occurs after the accretion stops because the light echo from the past AGN activity takes times to travel across the ISM of the host galaxy (e.g., Schawinski et al. 2015). In this period of the AGN-light echo, the AGN signature such as the X-ray emission at the center of the galaxy is not visible, but we can still observe the extended emission of the galaxy. Although O IIIB-4 and O IIIB-1 do not show any signature of the current AGN activity, it is still possible that their extended emission lines are the effect of the past AGN activity. If this is the case, O IIIB-4 and O IIIB-1 should show the AGN-like line ratios in the extended components.

We plot the blue diagram again in Figure 10 , but this time we separate the line ratios into 3 components based on the distance from the center of the [O III] blob (see also Section 4.6. For comparison purpose, we also plot the line ratios of the GPs at $z=0.1-0.4$. All components of O IIIB-4 show the $[\mathrm{O}$ III $] / \mathrm{H} \beta$ ratios consistent with the GPs and some fraction of the local star-forming galaxies that show the high $[\mathrm{O}$ III $] / \mathrm{H} \beta$ ratios. For O IIIB-1, the left component with the high $[\mathrm{O} \mathrm{III}] /[\mathrm{O} \mathrm{II}]$ ratio seen in the right panel of Figure 5 and in Figure 6 is located in the GP region. Therefore, it is unlikely that the extended [O III] emission lines of O IIIB-4 and O IIIB-1 are caused by the AGN photoionization left as the AGN-light echo after the super massive black hole at the center stops accreting. It is also seen from the figure that all components of $\mathrm{O}$ IIIB-2 lie in the intersect region of star-forming galaxies, composites, and AGNs. The line ratios of the central and right components of $\mathrm{O}$ IIIB-3 are consistent with those of AGNs, while the left component shows the lower $[\mathrm{O}$ II] $/ \mathrm{H} \beta$ ratio, closer to the star-forming galaxies and the GPs.

\subsection{Fast Radiative Shock}

Without the AGN contribution, the extended [O III] emission line and the gas outflow observed in O IIIB-4 and O IIIB-

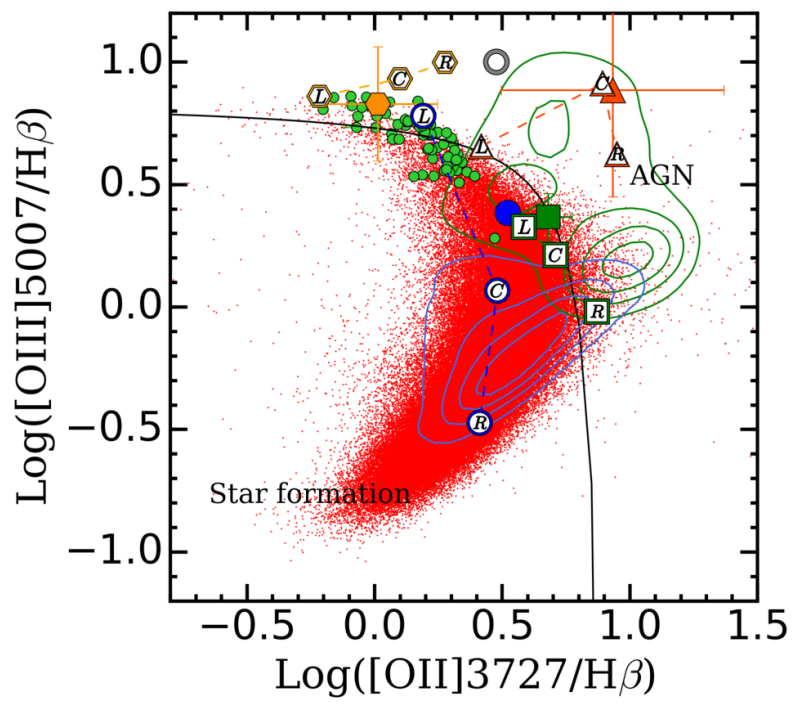

Figure 10. The blue diagram identical to the right panel of Figure 4 overplotted with three spatial components of the [O III] blobs. The letters " $\mathrm{L}$ ", " $\mathrm{C}$ ", and " $\mathrm{R}$ " correspond to the left, the center, and the right components of each blob. The circle, square, hexagon, and triangle represent O IIIB-1, O IIIB-2, O IIIB-3, and O IIIB-4, respectively. Small green circles are the green peas at $z=0.112-0.360$ by Cardamone et al. (2009). Red dots are the local SDSS star-forming galaxies. Blue and green contours represent composites and AGN, respectively.

1 are most likely fueled by the intense star formation activity (see also Section 4.7). However, some physical conditions are still required to explain such high $[\mathrm{O}$ III $] /[\mathrm{O}$ II $]$ ratios in the extended parts of the [O III] blobs. Supersonic motion of supernovae or gas outflows from a galaxy is able to create the radiative shock with the energy high enough to strongly affect the $\mathrm{H}$ II region and the ISM of the galaxy. A fast radiative shock, where extreme ultraviolet and soft X-ray photons are created by strong ionizing radiation behind the shock front, is one of the plausible explanations for the existence of the highly ionized emission lines (e.g., Dopita \& Sutherland 1996; Allen et al. 2008).

Allen et al. (2008) developed the library of radiative shock models called "MAPPINGS III," for the ISM with various ranges of metallicities (solar, Small and Large Magellanic Cloud metallicities), pre-shock densities of $0.01-1000 \mathrm{~cm}^{-3}$, and shock velocities of $100-1000 \mathrm{~km} \mathrm{~s}^{-1}$. When the velocity of the shock increases, the velocity of photoionization front increases and exceeds the shock velocities at a certain velocity limit $\left(v_{\text {shock }} \approx 170 \mathrm{~km} \mathrm{~s}^{-1}\right)$. At this point, the photoionization front is separated from the shock front and forms a "precursor" of the H II region, which dominates the optical emission of shocks at high shock velocities (Allen et al. 2008). In the models of shock+precursor, the $[\mathrm{O}$ III $] / \mathrm{H} \beta$ ratios become consistent with those of the extended components of the [O III] blobs; i.e., $\log (\mathrm{O} \mathrm{III}] / \mathrm{H} \beta) \geq 0.5$ (see Figure 10 , when the shock velocity reaches $v_{\text {shock }} \geq 350 \mathrm{~km} \mathrm{~s}^{-1}$ regardless of the values of the magnetic field and the metallicity. The prominent emission lines that is indicative of the fast radiative shocks include $\mathrm{He}$ II $\lambda 4686$ and [Ne v] $\lambda \lambda 3346,3426$ (e.g., Dopita \& Sutherland 1996, Thuan \& Izotov 2005; Izotov et al. 2012; Jaskot \& Oey 2013). However, we do not detect the He II or [Ne V] emission line in O IIIB-4 and O IIIB-1 above $3 \sigma$ flux limit of roughly $1.0 \times 10^{-18} \mathrm{erg} \mathrm{s}^{-1} \mathrm{~cm}^{-2}$. This places the upper limit of the $\mathrm{He} \mathrm{II} / \mathrm{H} \beta$ ratios to be $<0.03$ in the case of O IIIB-4. O IIIB-1 even seems to show the He II $\lambda 4686$ 


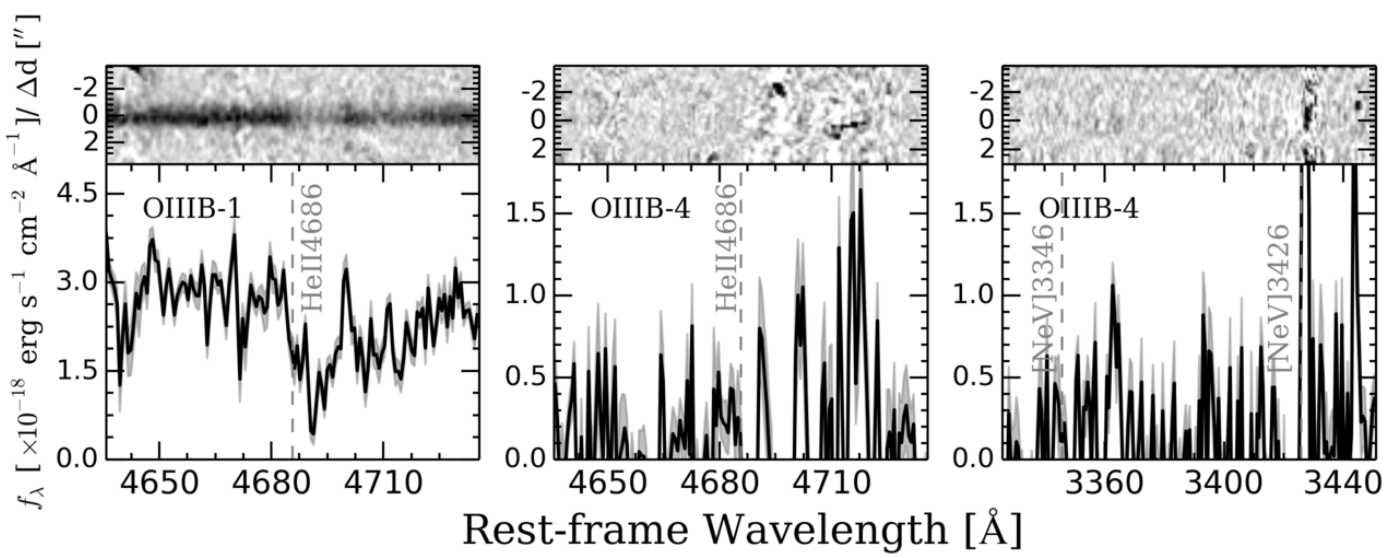

Figure 11. 1D and 2D spectra of O IIIB-1 (left panel) and O IIIB-4 (middle and right panels) at the wavelengths of He II $\lambda 4686$ and [Ne v] $\lambda \lambda 3346,3426$. The expected wavelength of each line is indicated with the gray dashed line.

as an absorption line (the left panel of Figure 11). The nondetection of He II and [Ne V] of O IIIB-4 is also shown in the middle and right panels of the figure, respectively. The lowest shock velocity of $100 \mathrm{~km} \mathrm{~s}^{-1}$ in the MAPPINGS III shock models by Allen et al. (2008) suggests the $\mathrm{He} \mathrm{II} / \mathrm{H} \beta$ line ratio of 0.47 assuming the density of $n=1.0 \mathrm{~cm}^{-3}$ and solar abundance.

For O IIIB-1 that we have Keck/MOSFIRE NIR spectrum, we also use another line diagnostics to investigate the shock scenario. Figure 12 shows the line ratios of each component of O IIIB-1 in various emission-line diagnostic diagram. The grids of both shock-only and shock+precursor models are also illustrated in the figure in the case of solar metallicity and preshock density of $n=1 \mathrm{~cm}^{-3}$. It is clearly seen that the left component of O IIIB-1 is not in the shock regions in all diagrams. Therefore, it is unlikely that the fast radiative shock is a physical process responsible for the high ionization parameters observed in O IIIB-4 and the extended part of O IIIB-1.

\subsection{Density Bounded Nebulae}

As already seen in Figure 5, the [O III]/[O II] ratio strongly depends on the ionization parameter and metallicity. The $[\mathrm{O} \mathrm{III}] /[\mathrm{O} \mathrm{II}]$ ratio increases with increasing ionization parameters and decreasing metallicities. In addition, Shirazi et al. (2014) argued that the high gas density of high-redshift galaxies affects the extreme value of [O III]/[O II] ratios. Nakajima \& Ouchi (2014) studied the ISM of star-forming galaxies at $z=0-3$ and found that the $[\mathrm{O} \mathrm{III}] /[\mathrm{O} \mathrm{II}]$ line ratio also depends on the escape fraction of the ionizing photons, which is defined as a ratio of ionizing photons escaping the galaxy to the totally produced ionizing photons. To explain the relationship between the $[\mathrm{O} \mathrm{III}] /[\mathrm{O}$ II $]$ ratio and the escape fraction, they classified star-forming nebulae into 2 types: ionization bounded and density bounded nebulae. The size of the ionization-bounded nebulae is equivalent to the Strömgren radius, which is determined by the ionization equilibrium between the producing rate of ionizing photons and the recombination rate. In the ionization bounded nebulae, highionization state of oxygen ions (producing [O III]) locates close to the ionizing sources, while the envelope of the $\mathrm{O}^{+}$ ions that produce $[\mathrm{O} \mathrm{II}]$ is around the edge of the nebulae (e.g, Shields 1990; Oey \& Kennicutt 1997; Pellegrini et al. 2012; Nakajima \& Ouchi 2014). On the other hand, some fraction of the ionizing photons is allowed to escape from the homogeneous density bounded nebulae. The $\mathrm{H}$ I clouds around the density bounded nebulae are so small that they are completely ionized. The radius of the density-bounded nebulae is thus constraint by the density of the gas cloud instead of the Strömgren radius, as the complete Strömgen sphere cannot be formed. The size of $\mathrm{O}^{+}$region is thus smaller in the density bounded nebulae than it is in the ionization bounded system. With similar size of the $\mathrm{O}^{2+}$ region, the density bounded nebulae would show larger $[\mathrm{O} \mathrm{III}] /[\mathrm{O} \mathrm{II}]$ ratio in the extended part.

The high [O III]/[O II] ratios of O IIIB-4 may be explained by the hypothesis that the [O III] blob is the density bounded nebula. The $[\mathrm{O} \mathrm{III}] /[\mathrm{O} \mathrm{II}]$ ratio in the outer region is due to the escaping ionizing photons produced by the intense star formation activity at the center of the blob. Arthur et al. (2011) performed the radiation-magnetohydrodynamic (MHD) simulation of the formation and expansion of the H II regions. They showed that the highly ionized density-bounded nebula is strongly influenced by the radiative feedback and gas instability, leading to the peculiar gas morphologies. O IIIB-1 has only one side with high [O III]/[O II] ratio, implying the asymmetric morphology of the outflow. However, it does not explain why the $[\mathrm{O} \mathrm{III}] /[\mathrm{O} \mathrm{II}]$ ratio increases at the greater distance from the center of O IIIB-1. Normally, the [O III]/[O II] ratio should decrease as a function of the distance from the center, as the $\mathrm{O}^{+}$ions increase while the $\mathrm{O}^{2+}$ ions decrease at the outer region (Pellegrini et al.2012). The studies of diffuse ionized gas (DIG) beyond the $\mathrm{H}$ II regions of the local galaxies typically show the decrease in $[\mathrm{O} \mathrm{III}] /[\mathrm{O} \mathrm{II}]$ ratios at the outer region of the galaxies and suggest the lower ionization parameters of DIG than those of the H II regions (e.g., Zhang et al. 2017). The higher [O III $] /[\mathrm{O}$ II $]$ ratio of the left component of O IIIB-1 than the rest of the galaxy seems to contradict with the typical DIG in the local universe. Nonetheless, Weber et al. (2019) performed the numerical analysis to predict the line ratios of $\mathrm{H}$ II regions and DIG by varying the metallicity, stellar effective temperature, and escape fraction $f_{\text {esc }}$. They found that the $[\mathrm{O} \mathrm{III}] /\left[\mathrm{O}_{\mathrm{II}}\right]$ ratios increase with radius only in the models of the density-bounded leaky $\mathrm{H}$ II regions with the stellar effective temperature of $40,000 \mathrm{~K}$ and the escape fraction less than $10 \%$. This might be the case for O IIIB1. O IIIB-1 may have the peculiar gas morphology that can cause the leakage of highly ionized photons through a hole in some direction away from the line of sight to the center of the galaxy. Dense gas clouds in the line of sight lead to the low escape fraction, whereas the [O III] emission is largely scattered toward us from the extended region (Bassett et al. 


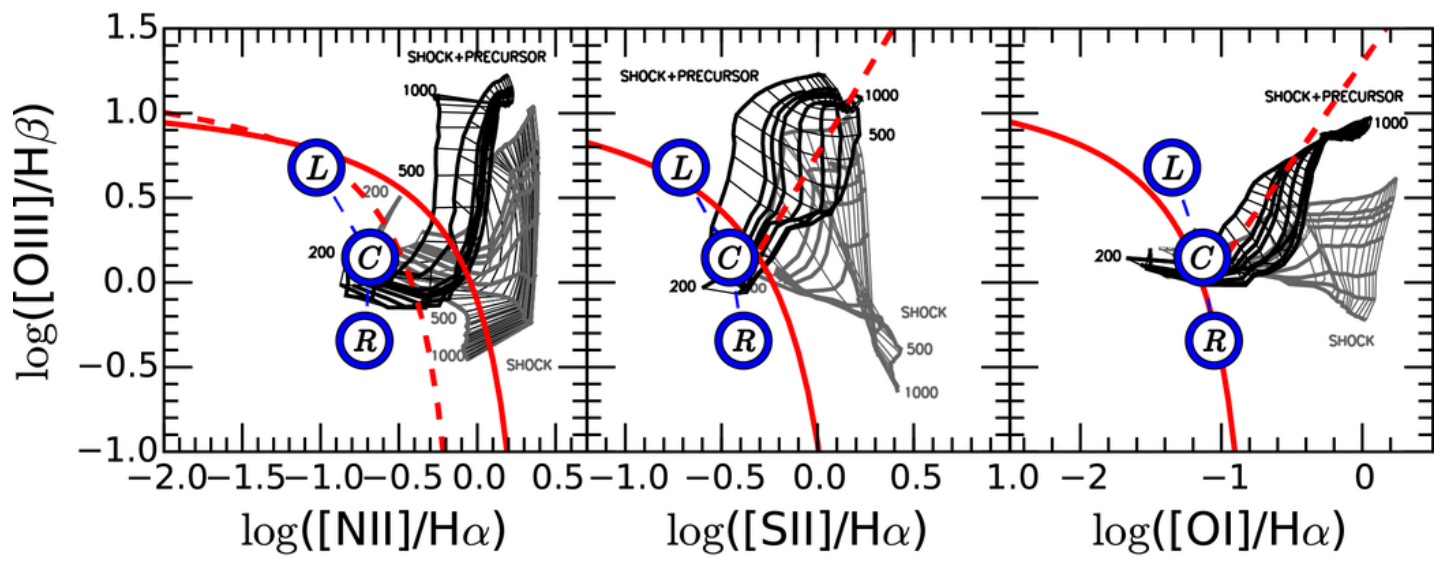

Figure 12. Emission-line diagnostic diagrams of O IIIB-1. Symbols for O IIIB-1 are identical to those in the previous figures. The gray and black grids show the shock-only and shock+precursor models with solar abundance and preshock density of $n=1 \mathrm{~cm}^{-3}$ obtained from MAPPINGS III (Allen et al. 2008), respectively. The grid consists of the lines of constant shock velocity in the range of $200-1000 \mathrm{~km} \mathrm{~s}^{-1}$ with $50 \mathrm{~km} \mathrm{~s}^{-1}$ intervals (thin lines) and the lines of constant magnetic parameter ranging from $B / n^{3 / 2}=0.0001 \mu \mathrm{G} \mathrm{cm}^{3 / 2}$ to $B / n^{3 / 2}=10.0 \mu \mathrm{G} \mathrm{cm}^{3 / 2}$ (thick lines).

2019). Further constraint on the escape fraction of O IIIB-1 is necessary to confirm this scenario.

\section{SUMMARY}

[O III] blobs are galaxies that exhibit the strong and spatially extended [O III] emission line beyond their stellar continuum. The first systematic survey of [O III] blobs has been conducted by Yuma et al. (2017). In this paper, we investigate the physical nature of the [O III] blobs in more details by using optical and near-infrared spectroscopy. We choose the spectroscopic targets based on their large extension of the [O III] emission line and their coordinates to maximize the number of targets per the observed mask. As a result, we observed two largest [O III] blobs at $z \sim 0.63$ (O IIIB-1 and O IIIB-2) and another two [O III] blobs at $z \sim 0.83$ (O IIIB-3 and $\mathrm{O}$ IIIB-4) in the optical wavelengths with Subaru/FOCAS. O IIIB-1 was also observed in the near-infrared wavelength with Keck/MOSFIRE. The main results are listed below.

1. We confirm the spectroscopic redshifts of all targets to be at the expected redshifts; i.e., O IIIB-1 and O IIIB-2 are at $z=0.6210$ and $z=0.6413$, respectively. O IIIB-3 and $\mathrm{O}$ IIIB-4 are at $z=0.8365$ and $z=0.8379$, respectively.

2. The original survey of [O III] blobs was based on the hypothesis that the spatially extended [O III] emission line is caused by the large-scale outflow of gas from the center of galaxies. We could confirm the outflow signature in O IIIB-1, which has the stellar continuum bright enough to be detected with the high $\mathrm{S} / \mathrm{N}$ ratios. The blueshifted Fe II $\lambda 2326$ absorption, Fe II* $\lambda 2626$ finestructure emission, and $\operatorname{Mg}$ II $\lambda 2804$ absorption lines are detected with the central velocity offsets of -160 $\mathrm{km} \mathrm{s}^{-1},-200 \mathrm{~km} \mathrm{~s}^{-1}$, and $-270 \mathrm{~km} \mathrm{~s}^{-1}$, respectively. The outflow velocities are slightly higher than those of normal star-forming galaxies at $z=1-2$ by Erb et al. (2012).

3. We examine AGN contribution by using the BPT diagram for O IIIB-1 with available NIR data and the $\left[\mathrm{O}_{\mathrm{III}}\right] / \mathrm{H} \beta$ vs. $[\mathrm{O} \mathrm{II}] / \mathrm{H} \beta$ diagram for the other three blobs, of which we have only the optical spectroscopic data. Among 4 observed [O III] blobs, O IIIB-3 is the only one that is heated by an AGN activity at the center based on its location on the $[\mathrm{O}$ III $] / \mathrm{H} \beta$ vs. $[\mathrm{O}$ II $] / \mathrm{H} \beta$ diagram. O IIIB-1, on the other hand, is located in the region consistent with the the pure star formation activity on the BPT diagram. O IIIB-4 is another [O III] blob that is identified as a normal star-forming galaxies in the blue diagram, though it shows a remarkably high $[\mathrm{O}$ III $] /[\mathrm{O}$ II $]$ line ratio. Lastly, we could not rule out the possibility of AGN contribution in O IIIB-2; it is likely to be a composite powered by both star formation and AGN activities.

4. The properties of the interstellar medium surrounding the $[\mathrm{O} \mathrm{III}]$ blobs have been examined by the [O III]/[O II]-R23 diagram. O IIIB-1, O IIIB-2, and O IIIB-3 show the [O III]/[O II] line ratios comparable to those of the local SDSS galaxies and the starforming galaxies at $z \sim 0.7$. Their inferred ionization parameters should be slightly less than $q_{i o n}=8 \times 10^{7}$ $\mathrm{cm} \mathrm{s}^{-1}$. Interestingly, O IIIB-4 exhibits the impressively high [O III $] /[\mathrm{O} \mathrm{II}]$ ratio. The line ratio and the $R 23$ index of O IIIB-4 agree well with those of the compact green peas at $z=0.1-0.4$. The ionization parameter of O IIIB-4 should be roughly in the order of $10^{8} \mathrm{~cm} \mathrm{~s}^{-1}$.

5. The radial profile of the $[\mathrm{O} \mathrm{III}] /[\mathrm{O} \mathrm{II}]$ ratios indicates that $\mathrm{O}$ IIIB-4 shows the $[\mathrm{O} \mathrm{III}] /[\mathrm{O} \mathrm{II}]$ ratio much greater than those of the star-forming galaxies at $z \sim 0.7$ and the other 3 [O III] blobs. The radial profile of O IIIB4 shows the almost constant [O III $] /[\mathrm{O}$ II] emissionline ratios of approximately $5-10$ over $14 \mathrm{kpc}$. The $[\mathrm{O} \mathrm{III}] /[\mathrm{O} \mathrm{II}]$ ratios are consistent with those of the GPs at $z=0.1-0.4$; however, the physical sizes are different as the GPs are compact with the [O III] emission lines within $5 \mathrm{kpc}$. Thus we call O IIIB-4 a giant green pea. Among the remaining 3 blobs, there is only one extended part of O IIIB-1 showing the [O III]/[O II] ratio as high as the GPs. It is suggested that such a high $[\mathrm{O} \mathrm{III}] /[\mathrm{O} \mathrm{II}]$ ratio found in the GPs at $z=0.1-0.4$ can also be found in the extended component beyond the stellar continuum of the galaxies as well.

6. The rest-frame [O III] equivalent width of O IIIB-4 is 
$845 \pm 27 \AA$. This is well in agreement with the typical GPs at $z=0.1-0.4$, which are the galaxies with high [O III] EWs by definition. The [O III] EWs of O IIIB-4 are still consistent with those of the GPs, whose [O III] EWs are in the range of $100-2000 \AA$, even if we divide the radial profile into 3 components according to the distance from the center of the blob. O IIIB-1, O IIIB2, and O IIIB-3 also have the rest-frame [O III] EWs in the same ranges as the GPs. When we divide the EWs into 3 components, the large [O III] EW of the left component of O IIIB-1 (>1000 $\AA$ ) is obviously seen. It is in agreement with the $[\mathrm{O} \mathrm{III}] /[\mathrm{O} \mathrm{II}]$ ratio and the $R 23$ index of this components that are similar to those of the GPs. However, the centres of O IIIB-1 and O IIIB-2 show the [O III] EWs of $\sim 100 \AA$ slightly smaller than those of the GPs.

7. The giant green pea, O IIIB-4, is also similar to green beans at $z \sim 0.3$ in that they show comparable [O III] EWs and consistent spatial extension of the [O III] emission line. However, unlike O IIIB-4, GBs is powered by AGN rather than star formation.

8. The giant GP, O IIIB-4, is the [O III] blob at $z \sim 0.83$ that has the low stellar mass of $7 \times 10^{7} M_{\odot}$, the high specific SFR of $2 \times 10^{2} \mathrm{Gyr}^{-1}$, and the low metallicity. Its physical properties are similar to those of the GPs found at $z=0.1-0.4$.

9. We check whether or not the [O III] extension of O IIIB4 and O IIIB-1 are an AGN-light echo influenced by the AGN activities in the past. The blue diagram suggests that the extended parts of O IIIB-4 and O IIIB-1 are caused by the star-forming activity. Thus we can rule out the AGN-light echo scenario.

10. The fast radiative shock is another potential mechanism to explain the high $[\mathrm{O}$ III $] /[\mathrm{O}$ II $]$ ratios. We do not detect the shock-indicative emission lines such as $[\mathrm{Ne} \mathrm{V}] \lambda \lambda 3346,3426$ and He II $\lambda 4686$ in O IIIB-4 and O IIIB- 1 at the $3 \sigma$ flux limit of $1.0 \times 10^{-18} \mathrm{erg} \mathrm{s}^{-1} \mathrm{~cm}^{-2}$. The upper limit of the $\mathrm{He} \mathrm{II} / \mathrm{H} \beta$ ratios $(<0.03)$ suggests that the fast radiative shock is unlikely.

The most likely scenario to explain the high [O III]/[O II] ratios observed in O IIIB-4 and the extended component of O IIIB-1 is probably density bounded nebulae. In the case of the density bounded nebulae, high ionization photons can escape from the center of the galaxy and the $\mathrm{O}^{+}$region producing [O II] emission line is small because of the small $\mathrm{H}$ I cloud. The density bounded region at the center might lead to a harder emitted spectrum that ionizes the more extended nebulae. Further observations are needed to confirm if the physical conditions of the [O III] blobs are really consistent with the density bounded nebulae.

The authors are very grateful to an anonymous referee for valuable comments that help improve the article. This work is supported by Faculty of Science, Mahidol University, Thailand and the Thailand Research Fund (TRF) through a research grant for new scholar (MRG6180279). We thank Moire Prescott for her useful comments. S.Y. thanks David J. Ruffolo for supports as a mentor of the TRF research grant. M.O. is supported by World Premier International Research Center Initiative (WPI Initiative), MEXT, Japan, and KAKENHI (15H02064, 17H01110, and 17H01114) Grant-in-Aid for Scientific Research (A) through Japan Society for the Promotion of Science.

\section{APPENDIX}

\section{A. CORRECTION FOR DUST ATTENUATION}

In order to obtain accurate results of the observed fluxes and the line ratios, we need to take dust extinction into account. For O IIIB-1 and O IIIB-2, we are able to detect the $\mathrm{H} \gamma$ emission lines and use the Balmer decrement to derive the dust attenuation. In addition, we can also detect the $\mathrm{H} \alpha$ emission line of $\mathrm{O}$ IIIB- 1 from the Keck/MOSFIRE spectrum. The observed $\mathrm{H} \gamma, \mathrm{H} \beta$, and $\mathrm{H} \alpha$ fluxes of O IIIB-1 are $4.92 \times 10^{-17} \mathrm{erg} \mathrm{s}^{-1} \mathrm{~cm}^{-2}, 1.24 \times 10^{-16} \mathrm{erg} \mathrm{s}^{-1} \mathrm{~cm}^{-2}$, and $4.76 \times 10^{-16} \mathrm{erg} \mathrm{s}^{-1} \mathrm{~cm}^{-2}$, respectively. These result in the $\mathrm{H} \gamma / \mathrm{H} \beta$ and $\mathrm{H} \alpha / \mathrm{H} \beta$ line ratios of $0.40 \pm 0.08$ and $3.84 \pm 0.40$, respectively. The $\mathrm{H} \gamma / \mathrm{H} \beta$ line ratio for $\mathrm{O}$ IIIB-2 is $0.39 \pm 0.26$ because the $\mathrm{H} \gamma$ and $\mathrm{H} \beta$ emission lines show the observed fluxes of $1.04 \times 10^{-16} \mathrm{erg} \mathrm{s}^{-1} \mathrm{~cm}^{-2}$, and $2.70 \times 10^{-16}$ $\mathrm{erg} \mathrm{s}^{-1} \mathrm{~cm}^{-2}$, respectively. The color excess $E(B-V)$ can be derived from the $\mathrm{H} \gamma / \mathrm{H} \beta$ ratio as follow.

$$
\begin{aligned}
E(B-V) & =\frac{E(H \beta-H \gamma)}{\kappa(H \beta)-\kappa(H \gamma)} \\
& =\frac{-2.5}{\kappa(H \beta)-\kappa(H \gamma)} \times \log _{10}\left[\frac{0.469}{(H \gamma / H \beta)_{\mathrm{obs}}}\right]
\end{aligned}
$$

Likewise, the $\mathrm{H} \alpha / \mathrm{H} \beta$ line ratio is related to the color excess as

$$
\begin{aligned}
E(B-V) & =\frac{E(H \beta-H \alpha)}{\kappa(H \beta)-\kappa(H \alpha)} \\
& =\frac{-2.5}{\kappa(H \beta)-\kappa(H \alpha)} \times \log _{10}\left[\frac{2.86}{(H \alpha / H \beta)_{\mathrm{obs}}}\right] .
\end{aligned}
$$

The intrinsic $\mathrm{H} \gamma / \mathrm{H} \beta$ and $\mathrm{H} \alpha / \mathrm{H} \beta$ ratios are 0.469 and 2.86, respectively. They are obtained by assuming Case B recombination with an electron density of $10^{2} \mathrm{~cm}^{-3}$ and temperature of $10^{4} \mathrm{~K}$ (Osterbrock 1989). The reddening curve $\kappa(\lambda)$ is derived by adopting the expression in Calzetti et al. (2000). Substituting $\kappa(\mathrm{H} \gamma)=5.12, \kappa(\mathrm{H} \beta)=4.60$, and $\kappa(\mathrm{H} \alpha)=3.33$ into the above equations, we obtain the color excesses $E(B-V)$ of $0.33 \pm 0.19$ mag and $0.24 \pm 0.10$ mag for O IIIB-1 as derived by using the 
Table 4

Color excesses of 4 [O III] blobs at $z=0.63-0.83$ dervied from the Balmer decrement and SED fitting method

\begin{tabular}{lrrr}
\hline \hline [O III] blobs & \multicolumn{2}{c}{$E(B-V)_{\text {nebular }}$} & $E(B-V)_{\text {stellar }}$ \\
& $\mathrm{H} \gamma / \mathrm{H} \beta$ & $\mathrm{H} \alpha / \mathrm{H} \beta$ & SED fitting \\
\hline O IIIB-1 & $0.33 \pm 0.19$ & $0.24 \pm 0.10$ & $0.18_{-0.04}^{+0.34}$ \\
O IIIB-2 & $0.41 \pm 0.42$ & - & $0.20_{-0.20}^{+0.40}$ \\
O IIIB-3 & - & - & $0.34_{-0.20}^{+0.36}$ \\
O IIIB-4 & - & - & $0.18_{-0.10}^{+0.28}$ \\
\hline
\end{tabular}

$\mathrm{H} \gamma / \mathrm{H} \beta$ and $\mathrm{H} \alpha / \mathrm{H} \beta$ line ratios, respectively. They are consistent with each other within $1 \sigma$ uncertainty. For O IIIB-1, we adopt $E(B-V)=0.33$ to correct for the dust attenuation. Because the $\mathrm{H} \gamma$ and $\mathrm{H} \beta$ lines are observed simultaneously with the same instrument, they are less suffered from different slit correction or flux calibration as compared to the $\mathrm{H} \alpha$ emission line. The color excess of $\mathrm{O}$ IIIB-2 estimated from the $\mathrm{H} \gamma / \mathrm{H} \beta$ ratio is $0.41 \pm 0.42$ mag. These nebular color excesses derived from the Balmer decrement are roughly consistent with those of stellar continuum estimated by the SED fitting method (Yuma et al. 2017) after multiplying by a factor of 0.44 described in Calzetti et al. (2000). For O IIIB-3 and O IIIB-4 whose the $\mathrm{H} \gamma$ emission lines are not detected, we estimate the nebular color excesses from the stellar color excesses obtained by the SED fitting method (Yuma et al. 2017). The color excesses of all four blobs are listed in Table 4. It is noteworthy that we assumed the constant dust extinction across the [O III] blobs. We checked the $\mathrm{H} \alpha / \mathrm{H} \beta$-ratio profile of $\mathrm{O}$ IIIB-1 and found that the $\mathrm{H} \alpha / \mathrm{H} \beta$ ratios decrease slightly with increasing distance up to $5-6 \mathrm{kpc}$ from the center of O IIIB-1. However, the ratios are still within the line-ratio uncertainty of the entire blob.

\section{REFERENCES}

Abazajian, K. N., Adelman-McCarthy, J. K., Agüeros, M. A., et al. 2009, ApJS, 182, 543

Aguirre, A., Dow-Hygelund, C., Schaye, J., \& Theuns, T. 2008, ApJ, 689, 851

Alexander, D. M., Swinbank, A. M., Smail, I., McDermid, R., \& Nesvadba, N. P. H. 2010, MNRAS, 402, 2211

Allen, M. G., Groves, B. A., Dopita, M. A., Sutherland, R. S., \& Kewley, L. J. 2008, ApJS, 178, 20

Amorín, R., Sommariva, V., Castellano, M., et al. 2014, A\&A, 568, L8

Amorín, R. O., Pérez-Montero, E., \& Vílchez, J. M. 2010, ApJL, 715, L128

Arthur, S. J., Henney, W. J., Mellema, G., de Colle, F., \&

Vázquez-Semadeni, E. 2011, MNRAS, 414, 1747

Baldwin, J. A., Phillips, M. M., \& Terlevich, R. 1981, PASP, 93, 5

Bassett, R., Ryan-Weber, E. V., Cooke, J., et al. 2019, MNRAS, 483, 5223

Bell, E. F., McIntosh, D. H., Katz, N., \& Weinberg, M. D. 2003, ApJS, 149, 289

Benson, A. J., Bower, R. G., Frenk, C. S., et al. 2003, ApJ, 599, 38

Bradshaw, E. J., Almaini, O., Hartley, W. G., et al. 2013, MNRAS

Brammer, G. B., van Dokkum, P. G., Illingworth, G. D., et al. 2013, ApJL, $765, \mathrm{~L} 2$

Bruzual, G. \& Charlot, S. 2003, MNRAS, 344, 1000

Calzetti, D., Armus, L., Bohlin, R. C., et al. 2000, ApJ, 533, 682

Cardamone, C., Schawinski, K., Sarzi, M., et al. 2009, MNRAS, 399, 1191

Cheung, E., Bundy, K., Cappellari, M., et al. 2016, Nature, 533, 504

Cicone, C., Maiolino, R., Sturm, E., et al. 2014, A\&A, 562, A21

Coil, A. L., Weiner, B. J., Holz, D. E., et al. 2011, ApJ, 743, 46

Dopita, M. A. \& Sutherland, R. S. 1996, ApJS, 102, 161

Elbaz, D., Daddi, E., Le Borgne, D., et al. 2007, A\&A, 468, 33

Erb, D. K., Quider, A. M., Henry, A. L., \& Martin, C. L. 2012, ApJ, 759, 26

Förster Schreiber, N. M., Genzel, R., Bouché, N., et al. 2009, ApJ, 706, 1364

Förster Schreiber, N. M., Genzel, R., Newman, S. F., et al. 2014, ApJ, 787, 38

Fotopoulou, S., Buchner, J., Georgantopoulos, I., et al. 2016, A\&A, 587, A142

Fumagalli, M., O’Meara, J. M., \& Prochaska, J. X. 2011, Sci, 334, 1245

Genzel, R., Newman, S., Jones, T., et al. 2011, ApJ, 733, 101

Harikane, Y., Ouchi, M., Yuma, S., et al. 2014, ApJ, 794, 129

Heckman, T. M., Armus, L., \& Miley, G. K. 1990, ApJS, 74, 833

Heckman, T. M., Lehnert, M. D., Strickland, D. K., \& Armus, L. 2000, ApJS, 129, 493

Ichikawa, K., Ueda, J., Bae, H.-J., et al. 2018, ArXiv e-prints

Izotov, Y. I., Thuan, T. X., \& Guseva, N. G. 2017, MNRAS, 471, 548

Izotov, Y. I., Thuan, T. X., \& Privon, G. 2012, MNRAS, 427, 1229

Jansen, F., Lumb, D., Altieri, B., et al. 2001, A\&A, 365, L1

Jaskot, A. E. \& Oey, M. S. 2013, ApJ, 766, 91

Kashikawa, N., Aoki, K., Asai, R., et al. 2002, PASJ, 54, 819

Kauffmann, G., Heckman, T. M., Tremonti, C., et al. 2003, MNRAS, 346, 1055

Kewley, L. J. \& Dopita, M. A. 2002, ApJS, 142, 35

Kewley, L. J., Dopita, M. A., Sutherland, R. S., Heisler, C. A., \& Trevena, J. 2001, ApJ, 556, 121
Kewley, L. J., Groves, B., Kauffmann, G., \& Heckman, T. 2006, MNRAS, 372,961

Kornei, K. A., Shapley, A. E., Martin, C. L., et al. 2012, ApJ, 758, 135

Lamareille, F. 2010, A\&A, 509, A53

Lilly, S. J., Carollo, C. M., Pipino, A., Renzini, A., \& Peng, Y. 2013, ApJ, 772,119

Lilly, S. J., Carollo, C. M., \& Stockton, A. N. 2003, ApJ, 597, 730

Lin, L., Lin, J.-H., Hsu, C.-H., et al. 2017, arXiv:1702.02464

Liu, G., Zakamska, N. L., Greene, J. E., Nesvadba, N. P. H., \& Liu, X. 2013, MNRAS, 430, 2327

Mannucci, F., Cresci, G., Maiolino, R., Marconi, A., \& Gnerucci, A. 2010, MNRAS, 408, 2115

Martin, C. L. 2005, ApJ, 621, 227

Martin, C. L., Shapley, A. E., Coil, A. L., et al. 2012, ApJ, 760, 127

McLean, I. S., Steidel, C. C., Epps, H. W., et al. 2012, in Proc. SPIE, Vol.

8446, Ground-based and Airborne Instrumentation for Astronomy IV, $84460 \mathrm{~J}$

Mutch, S. J., Croton, D. J., \& Poole, G. B. 2013, MNRAS, 435, 2445

Nakajima, K. \& Ouchi, M. 2014, MNRAS, 442, 900

Nakajima, K., Ouchi, M., Shimasaku, K., et al. 2013, ApJ, 769, 3

Nesvadba, N. P. H., Lehnert, M. D., De Breuck, C., Gilbert, A. M., \& van Breugel, W. 2008, A\&A, 491, 407

Newman, S. F., Genzel, R., Förster-Schreiber, N. M., et al. 2012a, ApJ, 761, 43

Newman, S. F., Shapiro Griffin, K., Genzel, R., et al. 2012b, ApJ, 752, 111

Noeske, K. G., Weiner, B. J., Faber, S. M., et al. 2007, ApJL, 660, L43

Oey, M. S. \& Kennicutt, Jr., R. C. 1997, MNRAS, 291, 827

Oke, J. B. \& Gunn, J. E. 1983, ApJ, 266, 713

Oppenheimer, B. D., Davé, R., Kereš, D., et al. 2010, MNRAS, 406, 2325

Osterbrock, D. E. 1989, S\&T, 78, 491

Pagel, B. E. J., Edmunds, M. G., Blackwell, D. E., Chun, M. S., \& Smith, G. 1979, MNRAS, 189, 95

Pellegrini, E. W., Oey, M. S., Winkler, P. F., et al. 2012, ApJ, 755, 40

Prochaska, J. X., Kasen, D., \& Rubin, K. 2011, ApJ, 734, 24

Ranalli, P., Koulouridis, E., Georgantopoulos, I., et al. 2016, A\&A, 590, A80

Rubin, K. H. R., Prochaska, J. X., Koo, D. C., et al. 2014, ApJ, 794, 156

Rupke, D. S., Veilleux, S., \& Sanders, D. B. 2005a, ApJS, 160, 87

-. 2005b, ApJS, 160, 115

Rupke, D. S. N., Gültekin, K., \& Veilleux, S. 2017, ApJ, 850, 40

Salpeter, E. E. 1955, ApJ, 121, 161

Schawinski, K., Koss, M., Berney, S., \& Sartori, L. F. 2015, MNRAS, 451, 2517

Schirmer, M., Diaz, R., Holhjem, K., Levenson, N. A., \& Winge, C. 2013, ApJ, 763, 60

Schirmer, M., Malhotra, S., Levenson, N. A., et al. 2016, MNRAS, 463, 1554

Shields, G. A. 1990, ARA\&A, 28, 525

Shirazi, M. \& Brinchmann, J. 2012, MNRAS, 421, 1043

Shirazi, M., Brinchmann, J., \& Rahmati, A. 2014, ApJ, 787, 120

Silk, J. \& Rees, M. J. 1998, A\&A, 331, L1

Simpson, C., Martínez-Sansigre, A., Rawlings, S., et al. 2006, MNRAS, 372,741

Simpson, C., Rawlings, S., Ivison, R., et al. 2012, MNRAS, 421, 3060 
Somerville, R. S., Hopkins, P. F., Cox, T. J., Robertson, B. E., \& Hernquist, L. 2008, MNRAS, 391, 481

Soto, K. T., Martin, C. L., Prescott, M. K. M., \& Armus, L. 2012, ApJ, 757, 86

Steidel, C. C., Erb, D. K., Shapley, A. E., et al. 2010, ApJ, 717, 289

Strüder, L., Briel, U., Dennerl, K., et al. 2001, A\&A, 365, L18

Sun, A.-L., Greene, J. E. \& Zakamska, N. L. 2017, ApJ, 835, 222

Thuan, T. X. \& Izotov, Y. I. 2005, ApJS, 161, 240

Tremonti, C. A., Heckman, T. M., Kauffmann, G., et al. 2004, ApJ, 613, 898

Turner, M. J. L., Abbey, A., Arnaud, M., et al. 2001, A\&A, 365, L27

Ueda, Y., Watson, M. G., Stewart, I. M., et al. 2008, ApJS, 179, 124

Ulrich, M.-H., Maraschi, L., \& Urry, C. M. 1997, ARA\&A, 35, 445 van de Voort, F., Schaye, J., Booth, C. M., \& Dalla Vecchia, C. 2011, MNRAS, 415, 2782

Veilleux, S. \& Osterbrock, D. E. 1987, ApJS, 63, 295

Weber, J. A., Pauldrach, A. W. A., \& Hoffmann, T. L. 2019, A\&A, 622, A115

Weiner, B. J., Coil, A. L., Prochaska, J. X., et al. 2009, ApJ, 692, 187

Yabe, K., Ohta, K., Iwata, I., et al. 2009, ApJ, 693, 507

Yang, H., Malhotra, S., Rhoads, J. E., \& Wang, J. 2017, ApJ, 847, 38

Yuma, S., Ouchi, M., Drake, A. B., et al. 2013, ApJ, 779, 53

-. 2017, ApJ, 841, 93

Zhang, K., Yan, R., Bundy, K., et al. 2017, MNRAS, 466, 3217 